\title{
Comparing 2HDM + scalar and pseudoscalar simplified models at LHC
}

\author{
Giorgio Arcadi, ${ }^{a, b}$ Giorgio Busoni, ${ }^{c}$ Thomas Hugle ${ }^{c}$ and Valentin Titus Tenorth ${ }^{c}$ \\ ${ }^{a}$ Dipartimento di Matematica e Fisica, Università di Roma Tre, \\ Via della Vasca Navale 84, 00146, Roma, Italy \\ ${ }^{b}$ INFN Sezione Roma Tre, \\ Roma, Italy \\ ${ }^{c}$ Max-Planck-Institut für Kernphysik, \\ Saupfercheckweg 1, 69117 Heidelberg, Germany \\ E-mail: giorgio.arcadi@uniroma3.it, giorgio.busoni@mpi-hd.mpg.de, \\ thugle@mpi-hd.mpg.de, tenorth@mpi-hd.mpg.de
}

ABSTRACT: In this work we compare the current experimental LHC limits of the 2HDM + scalar and pseudoscalar for the $t \bar{t}$, mono- $Z$ and mono- $h$ signatures and forecast the reach of future LHC upgrades for the mono- $Z$ channel. Furthermore, we comment on the possibility, in case of a signal detection, to discriminate between the two models. The $2 \mathrm{HDM}+\mathrm{S}$ and $2 \mathrm{HDM}+\mathrm{PS}$ are two notable examples of the so-called next generation of Dark Matter Simplified Models. They allow for a renormalizable coupling of fermionic, Standard Model singlet, Dark Matter with a two Higgs doublet sector, through the mixing of the latter with a scalar or pseudoscalar singlet.

Keywords: Beyond Standard Model, Higgs Physics

ARXIV EPRINT: 2001.10540 


\section{Contents}

1 Introduction 1

2 2HDM plus scalar/pseudoscalar: model description 3

2.1 Scalar potential 3

2.2 Mass spectrum and alignment limit $\quad 6$

2.3 Yukawa sector 8

2.4 Decay widths and branching ratios 9

3 Overview of model constraints 11

$\begin{array}{lll}3.1 & \text { Direct detection } & 12\end{array}$

$\begin{array}{lll}3.2 & \text { Indirect detection and relic density } & 12\end{array}$

$\begin{array}{lll}3.3 & \text { Flavour constraints } & 13\end{array}$

$\begin{array}{lll}3.4 & \text { Electroweak precision constraints } & 13\end{array}$

$\begin{array}{lll}3.5 & \text { Perturbativity and unitarity constraints } & 14\end{array}$

$\begin{array}{lll}3.6 & \text { Collider searches } & 15\end{array}$

$\begin{array}{lll}3.6 .1 t \bar{t}+\mathbb{E}_{T} & 15\end{array}$

$\begin{array}{lll}3.6 .2 \text { Mono-jet } & 15\end{array}$

4 Comparison of $2 \mathrm{HDM}+$ scalar/pseudoscalar and their LHC signatures 16

$\begin{array}{lll}4.1 \text { General aspects } & 16\end{array}$

$\begin{array}{lll}4.2 t \bar{t} \text { resonances } & 18\end{array}$

$\begin{array}{lll}4.3 & \text { Mono- } Z & 19\end{array}$

$\begin{array}{lll}\text { 4.3.1 Projected sensitivity } & 22\end{array}$

$\begin{array}{lll}4.4 \text { Mono- } h & 22\end{array}$

$\begin{array}{ll}4.5 & \text { Combined constraints } \\ \end{array}$

$\begin{array}{llr}5 & \text { Conclusions } & 26\end{array}$

$\begin{array}{ll}\text { A Formulae for the decay widths } & 28\end{array}$

$\begin{array}{lll}\text { A.1 Scalar model } & 28\end{array}$

$\begin{array}{lll}\text { A.2 Pseudoscalar model } & 30\end{array}$

\section{Introduction}

The search of Dark Matter (DM) has become since some years one of the primary objectives of the LHC collaboration and represents, as well, one of the main motivations for the proposal of new collider facilities. From a theoretical perspective, it is crucial to provide an efficient interface for the interpretation of the outcome of collider searches and to enforce the complementarity with the other DM search strategies, namely Direct and Indirect Detection. 
The so-called simplified models (see e.g. [1,2] for reviews) were born to satisfy this need. Their validity might be, however, questionable. The reason for this is twofold: on the one hand, their extreme simplicity might render these models overconstrained and on the other hand, associate them a very limited set of experimental signatures.

For example, given the fact that in these models the DM and the mediator are singlets with respect to the SM gauge group, the typical DM related collider signature is its pair production in association with initial state radiation (ISR). From the several considered candidates for ISR, like gluons, photons and $W, Z$ and Higgs bosons, the strongest constraints arise from gluon initial state induced mono-jet events. This holds particularly true for the (pseudo)scalar mediator under study in this work, since gluon fusion is typically the dominant production mechanism for them at the LHC and jets face the strongest SM gauge coupling. However, it is not expected that constraints from mono-jet searches significantly improve in the near future as the are already limited by systematic uncertainties and therefore do not benefit from only increasing the total accumulated luminosity [3-5]. Thus it is worth to investigate the potential constraints from mono- $Z / h$ which, on the contrary, are expected to improve significantly in the future LHC runs. On the theoretical side it is then necessary to elaborate appropriate models to interpret this kind of signals. In particular, the $Z$ or Higgs boson should not originate from ISR but rather be the products of a decaying mediator. This can naturally appear in models in which the mediator is not a complete SM singlet, which it also does not have to be from a theoretical perspective. Especially if the (pseudo)scalar mediator is supposed to couple to SM fermions in a gauge invariant way, it needs to be at least charged under the EW gauge group or coupled to the Higgs sector. Furthermore, as pointed out in e.g. [3, 6-13], simplified models that lack theoretical completions might potentially lead to unreliable predictions.

The second reason is the fact that these models often lack of a theoretical completion, rendering their predictions potentially not reliable (see e.g. [14-18]).

For this reason the community is actively looking for a new generation of DM models [8] which can account for a rich collider phenomenology and solid theoretical predictions still within a not too large set of free parameters.

The so called $2 \mathrm{HDM}+\mathrm{S}$ and $2 \mathrm{HDM}+\mathrm{PS}$ are two notable examples of this last category of models. First of all, they allow for a renormalizable coupling between a fermionic gauge singlet DM candidate and the Standard Model (SM). The DM couples, on first instance, with a (pseudo)scalar singlet. A portal with the SM sector is created by the mass mixing of the latter with the CP-even (CP-odd) states of a two doublet Higgs sector. The $2 \mathrm{HDM}+\mathrm{S}$ and $2 \mathrm{HDM}+\mathrm{PS}$ provide, moreover, a broad variety of collider signals, beyond simple missing energy signatures (see e.g. [3, 6-13]).

The aim of this paper is to study some of these particular signatures, namely $t \bar{t}$ resonances, mono- $Z$ and mono- $h$. Although, all of these signatures appear in both models, there can be a sizeable difference for the expected signal rates in the pseudoscalar and scalar model. Therefore, in our work we characterize the similarities and differences of the different signatures in the two models, by looking at the limits derived from current LHC data and analyses. In addition, we also look at the reach of the mono- $Z$ channel for future luminosities and comment on the possibility to discriminate between the $2 \mathrm{HDM}+\mathrm{S}$ and $2 \mathrm{HDM}+\mathrm{PS}$ models in case of a future signal detection. 
The paper is structured as follows. In the next section we will illustrate, from a theoretical perspective, the two models. Section 3 is, instead, devoted to a brief overview of their present phenomenological constraints. The results of our numerical study will be presented in section 4 . In section 5 we finally draw our conclusions.

\section{2HDM plus scalar/pseudoscalar: model description}

The models we analyse are $2 \mathrm{HDM}$ s containing an additional singlet scalar particle. These models have been widely discussed in litterature, e.g. [3, 7, 16, 19-22].

\subsection{Scalar potential}

The most general scalar potential we consider is

$$
V\left(\Phi_{1}, \Phi_{2}, S\right)=V_{2 \mathrm{HDM}}\left(\Phi_{1}, \Phi_{2}\right)+V_{S}(S)+V_{S 2 \mathrm{HDM}}\left(\Phi_{1}, \Phi_{2}, S\right)
$$

where $V_{2 \mathrm{HDM}}$ is the standard $2 \mathrm{HDM}$ potential, $V_{S}$ is the potential of the scalar singlet and $V_{S 2 \text { HDм }}$ is the potential involving interaction terms between the scalar singlet and the doublets. We start by reviewing the $2 \mathrm{HDM}$ part of the potential. In $2 \mathrm{HDMs}$, one has two doublets with identical charges, therefore having the freedom to choose a specific base $\Phi_{1}, \Phi_{2}$ in terms of which to write the potential. A generic $\mathrm{SU}(2)$ change of base,

$$
\left(\begin{array}{l}
\Phi_{1} \\
\Phi_{2}
\end{array}\right) \rightarrow\left(\begin{array}{l}
\Phi_{1}^{\prime} \\
\Phi_{2}^{\prime}
\end{array}\right)=U\left(\begin{array}{l}
\Phi_{1} \\
\Phi_{2}
\end{array}\right)
$$

where $U$ is an $\mathrm{SU}(2)$ matrix, will result in a potential giving the same physics but with different coefficients for the different terms. While it would be possible to get rid of residual freedom through basis independent methods, see e.g. [23-25], we will nevertheless consider, in this paper, two reference bases in which to write the potential. The first one is what we will call the flavour base. Assuming the Yukawa interactions of the $2 \mathrm{HDM}$ are either of type I, II, X, Y or Inert, the basis $\Phi_{1}, \Phi_{2}$ is defined in terms of which of the doublets interacts with the various fermions, and is therefore well defined. This basis is very useful to analyse the interactions of the scalars with fermions. The second basis we choose is the so-called Higgs basis, where one of the two doublets has no vacuum expectation value (vev). When writing the potential, we will label $\lambda_{i}, M_{i j}$ the coefficients of the potential in the flavour base, and we will use $\hat{\lambda}_{i}, \hat{M}_{i j}$ to label the coefficients of the potential in the Higgs basis. The doublets in the flavour basis will be labelled $\Phi_{1}, \Phi_{2}$, while the doublets in the Higgs basis will be labelled $\Phi_{h}, \Phi_{H}$. The $2 \mathrm{HDM}$ potential reads in the flavour basis

$$
\begin{aligned}
V_{2 \mathrm{HDM}}\left(\Phi_{1}, \Phi_{2}\right)= & M_{11}^{2} \Phi_{1}^{\dagger} \Phi_{1}+M_{22}^{2} \Phi_{2}^{\dagger} \Phi_{2}+\left(M_{12}^{2} \Phi_{2}^{\dagger} \Phi_{1}+\text { h.c. }\right)+\frac{\lambda_{1}}{2}\left(\Phi_{1}^{\dagger} \Phi_{1}\right)^{2}+\frac{\lambda_{2}}{2}\left(\Phi_{2}^{\dagger} \Phi_{2}\right)^{2} \\
& +\lambda_{3}\left(\Phi_{1}^{\dagger} \Phi_{1}\right)\left(\Phi_{2}^{\dagger} \Phi_{2}\right)+\lambda_{4}\left(\Phi_{2}^{\dagger} \Phi_{1}\right)\left(\Phi_{1}^{\dagger} \Phi_{2}\right)+\frac{1}{2}\left(\lambda_{5}\left(\Phi_{2}^{\dagger} \Phi_{1}\right)^{2}+\text { h.c. }\right),
\end{aligned}
$$

where a $\mathcal{Z}_{2}$ symmetry $\Phi_{1} \rightarrow \Phi_{1}, \Phi_{2} \rightarrow-\Phi_{2}$ has been imposed to suppress flavour changing neutral currents, thus removing the additional terms $\lambda_{6,7}$ containing three $\Phi_{1}$ doublets and one $\Phi_{2}$ doublet and vice versa. We still allow for the soft-breaking term $M_{12}^{2}$ that is 
necessary to have a scalar mass spectrum with the desired physical features. We also assume that the potential does not break CP explicitly. This means $\left(M_{12}^{2}\right)^{2}$ and $\lambda_{5}$ need to have the same phase (up to a negative relative sign), which can be reabsorbed into $\Phi_{2}$ by a field redefinition. Therefore we will assume that the parameters $\lambda_{i}, M_{i j}$ are all real. In this case, the $\mathrm{SU}(2)$ transformation freedom for the doublets reduces to $\mathrm{SO}(2)$ rotations:

$$
\left(\begin{array}{l}
\Phi_{1} \\
\Phi_{2}
\end{array}\right) \rightarrow\left(\begin{array}{c}
\Phi_{1}^{\prime} \\
\Phi_{2}^{\prime}
\end{array}\right)=\left(\begin{array}{cc}
\cos \beta & \sin \beta \\
-\sin \beta & \cos \beta
\end{array}\right)\left(\begin{array}{l}
\Phi_{1} \\
\Phi_{2}
\end{array}\right)
$$

The most general expressions for the other two terms in eq. (2.1) are

$$
\begin{aligned}
V_{S}(S)= & \frac{1}{2} M_{S S}^{2} S^{2}+\frac{1}{3} \mu_{S} S^{3}+\frac{1}{4} \lambda_{S} S^{4}, \\
V_{S 2 \mathrm{HDM}}\left(\Phi_{1}, \Phi_{2}, S\right)= & \mu_{11 S}\left(\Phi_{1}^{\dagger} \Phi_{1}\right) S+\mu_{22 S}\left(\Phi_{2}^{\dagger} \Phi_{2}\right) S+\left(\mu_{12 S} \Phi_{2}^{\dagger} \Phi_{1} S+\text { h.c. }\right) \\
& +\frac{\lambda_{11 S}}{2}\left(\Phi_{1}^{\dagger} \Phi_{1}\right) S^{2}+\frac{\lambda_{22 S}}{2}\left(\Phi_{2}^{\dagger} \Phi_{2}\right) S^{2}+\frac{1}{2}\left(\lambda_{12 S} \Phi_{2}^{\dagger} \Phi_{1} S^{2}+\text { h.c. }\right) .
\end{aligned}
$$

The $\mathcal{Z}_{2}$ symmetry might be also extended to these terms of the potential by defining suitable transformation properties for $S$. By choosing $S \rightarrow-S$ the terms $\mu_{S}, \mu_{11 S}, \mu_{22 S}$, would be forbidden, however they would still be allowed in case of soft-breaking of the $\mathcal{Z}_{2}$. The $\lambda_{12 S}$ term on the other hand is forbidden due to the assumed $\mathcal{Z}_{2}$ symmetry of the Higgs fields. All these terms would have, however, a negligible impact for the phenomenology discussed in this work, since they would affect only the scalar trilinear and quartic interactions. Consequently, in line with [3], we will not include them in our analysis.

In eq. (2.6), all coefficients need to be real, except for $\lambda_{12 S}$ and $\mu_{12 S}$. As we have set $\lambda_{12 S}=0$, the only remaining physical phase is the one of $\mu_{12 S}$. To conserve CP, this coefficient must be either purely real or imaginary (after rotating the $2 \mathrm{HDM}$ doublets to have all phases contained in the $2 \mathrm{HDM}$ potential reabsorbed). In the first case, mixing between the CP-even scalar states contained in the $\Phi_{1}$ and $\Phi_{2}$ doubles and the singlet scalar would be obtained. In the latter case instead, the singlet state would mix with the neutral CP-odd states contained in the doublets.

The $2 \mathrm{HDM}+\mathrm{PS}$, defined in [19], and discussed in [3], is obtained using the latter option. Relabeling $S$ to $P$ to evidence more explicitly its CP-odd nature, the resulting potential is

$$
V\left(\Phi_{1}, \Phi_{2}, P\right)=V_{2 \mathrm{HDM}}\left(\Phi_{1}, \Phi_{2}\right)+V_{P}(P)+V_{P 2 \mathrm{HDM}}\left(\Phi_{1}, \Phi_{2}, P\right)
$$

where $V_{2 \mathrm{HDM}}$ is the one given in eq. (2.3) while

$$
\begin{aligned}
V_{P}(P) & =\frac{1}{2} M_{P P}^{2} P^{2}+\frac{1}{4} \lambda_{P} P^{4}, \\
V_{P 2 \mathrm{HDM}}\left(\Phi_{1}, \Phi_{2}, P\right) & =\frac{\lambda_{11 P}}{2}\left(\Phi_{1}^{\dagger} \Phi_{1}\right) P^{2}+\frac{\lambda_{22 P}}{2}\left(\Phi_{2}^{\dagger} \Phi_{2}\right) P^{2}+\mu_{12 P} P\left(i \Phi_{1}^{\dagger} \Phi_{2}+\text { h.c. }\right),
\end{aligned}
$$

with

$$
\Phi_{i}=\left(\begin{array}{c}
\Phi_{i}^{+} \\
\frac{v_{i}+\rho_{i}+i \eta_{i}}{\sqrt{2}}
\end{array}\right), \quad P=\eta_{3},
$$


and $v_{1,2}$ are usually parametrized in terms of

$$
\tan \beta=\frac{v_{2}}{v_{1}}, \quad \text { with } \quad v_{1}^{2}+v_{2}^{2}=v^{2} .
$$

$\rho_{1,2}$ are CP-even scalar particles, and $\eta_{1,2,3}$ are CP-odd scalar particles, but not necessarily mass eigenstates.

It is convenient to rotate $\left\{\Phi_{1}, \Phi_{2}\right\}$ to the Higgs basis $\left\{\Phi_{h}, \Phi_{H}\right\}$, where $\left\langle\Phi_{H}\right\rangle=0$ and $\left\langle\Phi_{h}\right\rangle=v \approx 246 \mathrm{GeV}$. The potential then reads

$$
\hat{V}\left(\Phi_{h}, \Phi_{H}, P\right)=\hat{V}_{2 \mathrm{HDM}}\left(\Phi_{h}, \Phi_{H}\right)+\hat{V}_{P}(P)+\hat{V}_{P 2 \mathrm{HDM}}\left(\Phi_{h}, \Phi_{H}, P\right),
$$

where

$$
\begin{aligned}
\hat{V}_{2 \mathrm{HDM}}\left(\Phi_{h}, \Phi_{H}\right)= & \hat{M}_{h h}^{2} \Phi_{h}^{\dagger} \Phi_{h}+\hat{M}_{H H}^{2} \Phi_{H}^{\dagger} \Phi_{H}+\left(\hat{M}_{h H}^{2} \Phi_{H}^{\dagger} \Phi_{h}+\text { h.c. }\right) \\
& +\frac{\hat{\lambda}_{h}}{2}\left(\Phi_{h}^{\dagger} \Phi_{h}\right)^{2}+\frac{\hat{\lambda}_{H}}{2}\left(\Phi_{H}^{\dagger} \Phi_{H}\right)^{2} \\
& +\hat{\lambda}_{3}\left(\Phi_{h}^{\dagger} \Phi_{h}\right)\left(\Phi_{H}^{\dagger} \Phi_{H}\right)+\hat{\lambda}_{4}\left(\Phi_{H}^{\dagger} \Phi_{h}\right)\left(\Phi_{h}^{\dagger} \Phi_{H}\right)+\frac{\hat{\lambda}_{5}}{2}\left(\left(\Phi_{H}^{\dagger} \Phi_{h}\right)^{2}+\text { h.c. }\right) \\
& +\hat{\lambda}_{6} \Phi_{h}^{\dagger} \Phi_{h}\left(\left(\Phi_{H}^{\dagger} \Phi_{h}\right)^{2}+h . c .\right)+\hat{\lambda}_{7} \Phi_{H}^{\dagger} \Phi_{H}\left(\left(\Phi_{H}^{\dagger} \Phi_{h}\right)^{2}+\text { h.c. }\right), \\
\hat{V}_{P}(P)= & \frac{1}{2} \hat{M}_{P P}^{2} P^{2}+\frac{\hat{\lambda}_{P}}{4} P^{4}, \\
\hat{V}_{P 2 \mathrm{HDM}}\left(\Phi_{h}, \Phi_{H}, P\right)= & \frac{\hat{\lambda}_{H H P}}{2} \Phi_{H}^{\dagger} \Phi_{H} P^{2}+\frac{\hat{\lambda}_{h h P}}{2} \Phi_{h}^{\dagger} \Phi_{h} P^{2}+\frac{\hat{\lambda}_{h H P}}{2} P^{2}\left(\Phi_{H}^{\dagger} \Phi_{h}+\text { h.c. }\right) \\
& +\mu_{12 P} P\left(i \Phi_{h}^{\dagger} \Phi_{H}+h . c .\right) .
\end{aligned}
$$

The two Higgs doublets are then defined by

$$
\begin{gathered}
\Phi_{h}=\cos \beta \Phi_{1}+\sin \beta \Phi_{2}=\left(\begin{array}{c}
G^{+} \\
\frac{v+\hat{\rho}_{1}+i G^{0}}{\sqrt{2}}
\end{array}\right), \\
\Phi_{H}=-\sin \beta \Phi_{1}+\cos \beta \Phi_{2}=\left(\begin{array}{c}
H^{+} \\
\frac{\hat{\rho}_{2}+i \hat{\rho}_{3}}{\sqrt{2}}
\end{array}\right),
\end{gathered}
$$

where $G^{ \pm}, G^{0}$ are the SM Goldstone bosons, and $H^{ \pm}$is the $2 \mathrm{HDM}$ charged scalar. All these particles are mass eigenstates. The particles $\hat{\rho}_{1,2}$ are CP-even scalars that are linear combinations of the standard model Higgs boson $h$ and an additional heavy scalar $H . \hat{\rho}_{3}, \eta_{3}$ are, instead, CP-odd and their combination will originate mass eigenstates which will be labelled $a$ and $A$ with, in general, $M_{a}<M_{A}$.

Note that, in general, the terms $\lambda_{6}, \lambda_{7}, \lambda_{h H P}$ arise when changing base. Moreover, the coefficient of the term $P\left(i \Phi_{h}^{\dagger} \Phi_{H}+\right.$ h.c. $)$ does not change when changing base.

For the scalar case, it is possible to proceed in the same way, assuming $\mu_{12 S}$ to be purely real. However, in the case of the scalar, there is also another way to obtain a mixing between the singlet and the CP-even scalars of the doublets. One can in fact assume that the singlet develops a vev. This is the approach taken by $[7,16]$. In this approach, one assumes that the scalar potential has a spontaneously broken $\mathcal{Z}_{2}$ symmetry w.r.t. which 
only the particle $S$ is odd, that gets rid of all cubic terms, independently of the basis in which the potential is written in. This may arise naturally, for example, in the case where $S$ is part of a complex scalar charged under a dark U(1) gauge group. In [7], the authors write the potential directly in the Higgs basis as

$$
\hat{V}\left(\Phi_{h}, \Phi_{H}, S\right)=\hat{V}_{2 \mathrm{HDM}}\left(\Phi_{h}, \Phi_{H}\right)+\hat{V}_{S}(S)+\hat{V}_{S 2 \mathrm{HDM}}\left(\Phi_{h}, \Phi_{H}, S\right),
$$

where

$$
\begin{aligned}
\hat{V}_{2 \mathrm{HDM}}\left(\Phi_{h}, \Phi_{H}\right)= & \hat{M}_{h h}^{2} \Phi_{h}^{\dagger} \Phi_{h}+\hat{M}_{H H}^{2} \Phi_{H}^{\dagger} \Phi_{H}+\left(\hat{M}_{h H}^{2} \Phi_{H}^{\dagger} \Phi_{h}+\text { h.c. }\right) \\
& +\frac{\hat{\lambda}_{h}}{2}\left(\Phi_{h}^{\dagger} \Phi_{h}\right)^{2}+\frac{\hat{\lambda}_{H}}{2}\left(\Phi_{H}^{\dagger} \Phi_{H}\right)^{2} \\
& +\hat{\lambda}_{3}\left(\Phi_{h}^{\dagger} \Phi_{h}\right)\left(\Phi_{H}^{\dagger} \Phi_{H}\right)+\hat{\lambda}_{4}\left(\Phi_{H}^{\dagger} \Phi_{h}\right)\left(\Phi_{h}^{\dagger} \Phi_{H}\right)+\frac{\hat{\lambda}_{5}}{2}\left(\left(\Phi_{H}^{\dagger} \Phi_{h}\right)^{2}+\text { h.c. }\right), \\
\hat{V}_{S}(S)= & \frac{1}{2} \hat{M}_{S S}^{2} S^{2}+\frac{\hat{\lambda}_{S}}{4} S^{4}, \\
\hat{V}_{S 2 \mathrm{HDM}}\left(\Phi_{h}, \Phi_{H}, S\right)= & \frac{\hat{\lambda}_{H H S}}{2} \Phi_{H}^{\dagger} \Phi_{H} S^{2}+\frac{\hat{\lambda}_{h h S}}{2} \Phi_{h}^{\dagger} \Phi_{h} S^{2}+\frac{1}{2}\left(\hat{\lambda}_{h H S} \Phi_{H}^{\dagger} \Phi_{h} S^{2}+\text { h.c. }\right),
\end{aligned}
$$

with

$$
\begin{aligned}
\Phi_{h} & =\left(\begin{array}{c}
G^{+} \\
\frac{v+\hat{\rho}_{1}+i G^{0}}{\sqrt{2}}
\end{array}\right), \\
\Phi_{H} & =\left(\begin{array}{c}
H^{+} \\
\frac{\hat{\rho}_{2}+i A}{\sqrt{2}}
\end{array}\right), \\
S & =v_{s}+\hat{\rho}_{3},
\end{aligned}
$$

this time, on top of the SM Goldstone bosons $G$ and the charged scalar $H^{ \pm}$, there is a single CP-odd scalar, $A$, and three CP-even scalars, $\hat{\rho}_{1,2,3}$, that mix together, and are linear combinations of $h$ and $S_{1,2}$, which are two new CP-even scalar particles.

Note that, in this potential written in the Higgs base, the terms $\hat{\lambda}_{6,7}$ are absent, contrary to the pseudoscalar case, where changing to the Higgs base switches these terms on, in general.

\subsection{Mass spectrum and alignment limit}

The $M_{h}=125 \mathrm{GeV}$ Higgs boson is experimentally observed to be very similar to the SM one. To avoid most of the constraints from Higgs physics, we choose to work in the so called alignment limit, i.e. we impose specific relations among the parameters of the scalar potential such that the mixing between the $\hat{\rho}_{1}$ and $\hat{\rho}_{2}$ is negligible and $\hat{\rho}_{1}$ is always identified as the experimentally observed $125 \mathrm{GeV}$ SM-like CP-even Higgs.

In the pseudoscalar model, the alignment limit can be achieved by assuming a specific value for $\beta$ :

$$
\cos 2 \beta=-\frac{\lambda_{1}-\lambda_{2}}{\lambda_{1}+\lambda_{2}-2\left(\lambda_{3}+\lambda_{4}+\lambda_{5}\right)}
$$


Alternatively, one can assume the presence of the CP2 symmetry [26], that gives the following relations between the parameters

$$
\lambda_{1}=\lambda_{2}=\lambda_{3}+\lambda_{4}+\lambda_{5}
$$

In the latter approach, one has the advantage that, when switching base, one has $\lambda_{i}=\hat{\lambda}_{i}$ for $i=1,2,3,4,5$ and $\hat{\lambda}_{6,7}=0$.

In case of the scalar model, one also needs the additional assumption

$$
\hat{\lambda}_{h h S}=0
$$

This additional condition arises because of the different choice on how the mixing is achieved, i.e. with non-zero vev for $S$.

Assuming either eq. (2.25) or (2.26), the mass matrix of the model is block diagonal. In the pseudoscalar model, it is made of a diagonal block containing two zero eigenvalues, corresponding to the Goldstone bosons $G^{0}, G^{ \pm}$, and the masses $M_{h}^{2}, M_{H^{ \pm}}^{2}, M_{H}^{2}$, plus a two-dimensional block. Diagonalizing the block, one gets the masses and the mixing angle of the 2 CP-odd scalars

$$
\sin 2 \theta=\frac{2 v \mu_{12 P}}{M_{A}^{2}-M_{a}^{2}} .
$$

In our convention, similar to other authors [3, 7], we are concentrating on the case $M_{a}<M_{A}$. The original set of parameters $\lambda_{1,2,4,5}, M_{11}^{2}, M_{22}^{2}, M_{12}^{2}, M_{P P}^{2}, \mu_{12 P}$ can therefore be expressed in terms of $M_{h}, M_{H}, M_{H^{ \pm}}, M_{A}, M_{a}, \theta, \tan \beta, v$ together with the alignment condition. The parameters $\lambda_{3}, \lambda_{11 P}, \lambda_{22 P}, \lambda_{P}$ remain free if the alignment condition eq. (2.25) is chosen, while when choosing eq. (2.26) only $\lambda_{11 P}, \lambda_{22 P}, \lambda_{P}$ remain free, while the value of $\lambda_{3}$ gets fixed.

In the scalar model instead, the mass matrix is made of a diagonal block containing two zero eigenvalues, corresponding to the Goldstone bosons $G^{0}, G^{ \pm}$, and the masses $M_{h}^{2}, M_{H^{ \pm}}^{2}, M_{A}^{2}$, plus a two-dimensional block. Diagonalizing the block, one gets the masses and the mixing angle of the $2 \mathrm{CP}$-even scalars

$$
\sin 2 \theta=\frac{2 \hat{\lambda}_{h H s} v v_{S}}{M_{S_{1}}^{2}-M_{S_{2}}^{2}}
$$

Also for the scalar case, we will concentrate on the case $M_{S_{2}}<M_{S_{1}}$. Again, one can exchange the set of parameters $\hat{\lambda}_{1,2,4,5}, M_{11}^{2}, M_{22}^{2}, M_{12}^{2}, M_{S S}^{2}, \mu_{12 S}$ for the set $M_{h}, M_{S_{1}}$, $M_{H^{ \pm}}, M_{A}, M_{S_{2}}, \theta, \tan \beta, v$, together with the alignment condition. The additional alignment condition eq. (2.27) sets $\hat{\lambda}_{11 S}$. When choosing alignment condition eq. (2.25), the parameters $\hat{\lambda}_{3}, \hat{\lambda}_{22 S}, \lambda_{S}$ remain free, while when choosing alignment condition eq. (2.26), only $\hat{\lambda}_{22 S}, \lambda_{S}$ remain free.

To avoid having the couplings $\lambda_{i}$ varying in an uncontrolled way when varying $\tan \beta$, throughout the rest of the paper we will adopt the alignment condition eq. (2.26), together with the mass degenerate assumption $M_{H}=M_{H^{ \pm}}=M_{A}>M_{a}$ or $M_{A}=M_{H^{ \pm}}=M_{S_{1}}>M_{S_{2}}$, respectively for the pseudoscalar/scalar model. Moreover, to get a reasonable comparison 
between the pseudoscalar and the scalar $\operatorname{model}^{1}$ and to avoid variations with $\tan \beta$, we decide to set

$$
\begin{aligned}
& \hat{\lambda}_{h h S}=\hat{\lambda}_{H H S}=0, \\
& \hat{\lambda}_{h h P}=\hat{\lambda}_{H H P}=0,
\end{aligned}
$$

or equivalently

$$
\begin{aligned}
& \lambda_{11 S}=\lambda_{22 S}=0, \\
& \lambda_{11 P}=\lambda_{22 P}=0,
\end{aligned}
$$

for the rest of the paper. This is most relevant for the $h A a$ vertex $\left(h S_{1} S_{2}\right.$ vertex for the scalar model), which appears in mono- $h$ processes and depends on

$$
\begin{aligned}
& \hat{\lambda}_{h h S}=\lambda_{11 S} \cos ^{2} \beta+\lambda_{22 S} \sin ^{2} \beta, \\
& \hat{\lambda}_{h h P}=\lambda_{11 P} \cos ^{2} \beta+\lambda_{22 P} \sin ^{2} \beta .
\end{aligned}
$$

Finally, the parameter $\lambda_{P, S}$ is not relevant for our signatures.

\subsection{Yukawa sector}

The Yukawa interactions of the SM fermions with the Higgs doublets can be expressed as

$$
L_{\text {Yukawa }}=-\sum_{n=1,2}\left(Y_{n, i j}^{u} \bar{Q}_{L}^{i} u_{R}^{j} \widetilde{\Phi}_{n}+Y_{n, i j}^{d} \bar{Q}_{L}^{i} d_{R}^{j} \Phi_{n}+Y_{n, i j}^{l} \bar{L}_{L}^{i} l_{R}^{j} \Phi_{n}+\text { h.c. }\right) .
$$

As in standard 2HDMs, we shall need to choose Yukawa structures that keep potentially dangerous flavour violating processes under control. We outline the possibilities below, and explore the DM phenomenology of these choices in section 3.1 by determining direct detection constraints.

Rewriting eq. (2.36) in the Higgs basis we have

$$
L_{\text {Yukawa }}=-\sum_{n=h, H}\left(Y_{n, i j}^{u} \bar{Q}_{L}^{i} u_{R}^{j} \widetilde{\Phi}_{n}+Y_{n, i j}^{d} \bar{Q}_{L}^{i} d_{R}^{j} \Phi_{n}+Y_{n, i j}^{l} \bar{L}_{L}^{i} l_{R}^{j} \Phi_{n}+\text { h.c. }\right),
$$

where the matrices $Y_{h, i j}^{u, d, l}$ have to be the SM Yukawa matrices, namely

$$
Y_{h}^{i} \equiv Y_{\mathrm{SM}}^{i},
$$

while the Yukawa matrices of the additional doublet are assumed to be proportional to the SM ones

$$
Y_{H}^{i}=\epsilon_{i} Y_{\mathrm{SM}}^{i}
$$

where the $\epsilon_{i}$ are Yukawa scaling factors, with $i=u, d, l$. This Yukawa structure is the so-called Aligned Yukawa model [27-33], which satisfies Natural Flavour Conservation. In special cases where the $\epsilon_{i}$ satisfy certain relationships, the Aligned Yukawa structure can

\footnotetext{
${ }^{1}$ We remind the reader that the scalar model has the additional alignment constraint eq. (2.27) that sets $\hat{\lambda}_{11 S}$.
} 


\begin{tabular}{|c|c|c|c|}
\hline Model & $\epsilon_{d}$ & $\epsilon_{u}$ & $\epsilon_{l}$ \\
\hline Type I & $\cot \beta$ & $\cot \beta$ & $\cot \beta$ \\
Type II & $-\tan \beta$ & $\cot \beta$ & $-\tan \beta$ \\
Type X & $\cot \beta$ & $\cot \beta$ & $-\tan \beta$ \\
Type Y & $-\tan \beta$ & $\cot \beta$ & $\cot \beta$ \\
Inert & 0 & 0 & 0 \\
\hline
\end{tabular}

Table 1. Values of the coefficients $\epsilon_{u, d, l}$ which correspond to models with discrete $\mathcal{Z}_{2}$ symmetries.

correspond to one of the $\mathcal{Z}_{2}$ symmetric Yukawa structures (type I, II, X or Y), as shown in table 1. As we will only probe values of $\tan \beta \leq 3$, our constraints will be valid for all Yukawa structures included in table 1, except the Inert one.

The mass spectrum of the model contains an additional fermion, the DM candidate. Assuming it to be a SM singlet, it will couple, in the interactions basis, only with the scalar,

$$
\mathcal{L}_{\mathrm{DM}}=-y_{\chi}^{S} S \bar{\chi} \chi
$$

or the pseudoscalar singlet

$$
\mathcal{L}_{\mathrm{DM}}=-y_{\chi}^{P} P \bar{\chi} \gamma^{5} \chi
$$

Due to the mass mixing between the $\mathrm{SU}(2)$ singlet and doublet scalars, the DM will in any case couple to the CP-even or CP-odd physical states according to whether we will consider the $2 \mathrm{HDM}+\mathrm{S}$ or $2 \mathrm{HDM}+\mathrm{PS}$ model.

Type I, II, $\mathbf{X}$ and $\mathbf{Y}$. To suppress flavour-changing neutral currents in $2 \mathrm{HDM}$, it is possible to assume the presence of a $\mathcal{Z}_{2}$ symmetry on the Yukawa sector, allowing only one of the two doublets $\Phi_{1,2}$ to couple to a certain type of quarks and leptons. This hypothesis goes under the name of natural flavour conservation (NFC). The presence of the charged scalar $H^{ \pm}$still allows FCNC to appear at loop level. Loop generated FCNC allow to set limits on $\tan \beta$ and the charged scalar mass also in the case of Higgs-alignment, that is weakly constrained by many other Higgs physics observables.

Each possible assignment of the $\mathcal{Z}_{2}$ charges results in a different type of $2 \mathrm{HDM}$. These types are listed in table 1.

\subsection{Decay widths and branching ratios}

In this section we compare the branching ratios (BR) of the four neutral spin-0 states in the $2 \mathrm{HDM}+\mathrm{PS}$ and $2 \mathrm{HDM}+\mathrm{S}$ for the later discussion. We give analytic expressions of the dominant decay widths for those and the charged scalars in appendix A. For all plots and interpretations we used the parameter values from eq. (4.4) and fixed $M_{A}=500 \mathrm{GeV}$ and $\tan \beta=1$ such that the findings are applicable to all types of $2 \mathrm{HDMs}$ (besides the Inert one). 

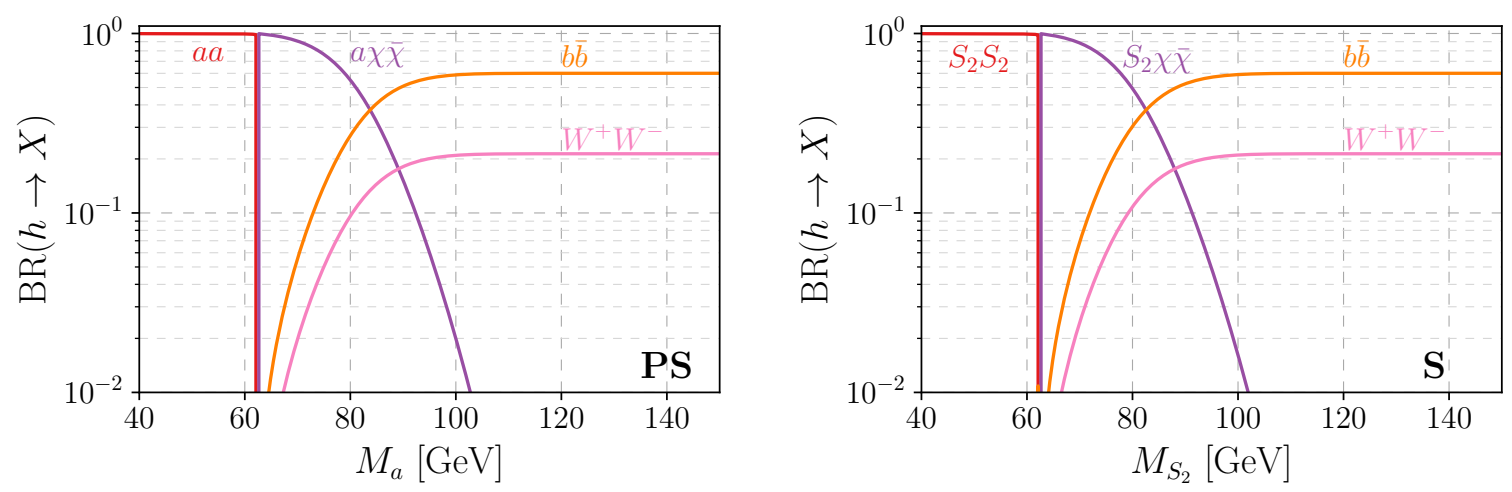

Figure 1. Dominant branching ratios of the SM Higgs-like scalar $h$ for $\tan \beta=1, m_{\chi}=10 \mathrm{GeV}$ and other parameter values as given in eq. (4.4) in the $2 \mathrm{HDM}+\mathrm{PS}$ (left) and $2 \mathrm{HDM}+\mathrm{S}$ (right).
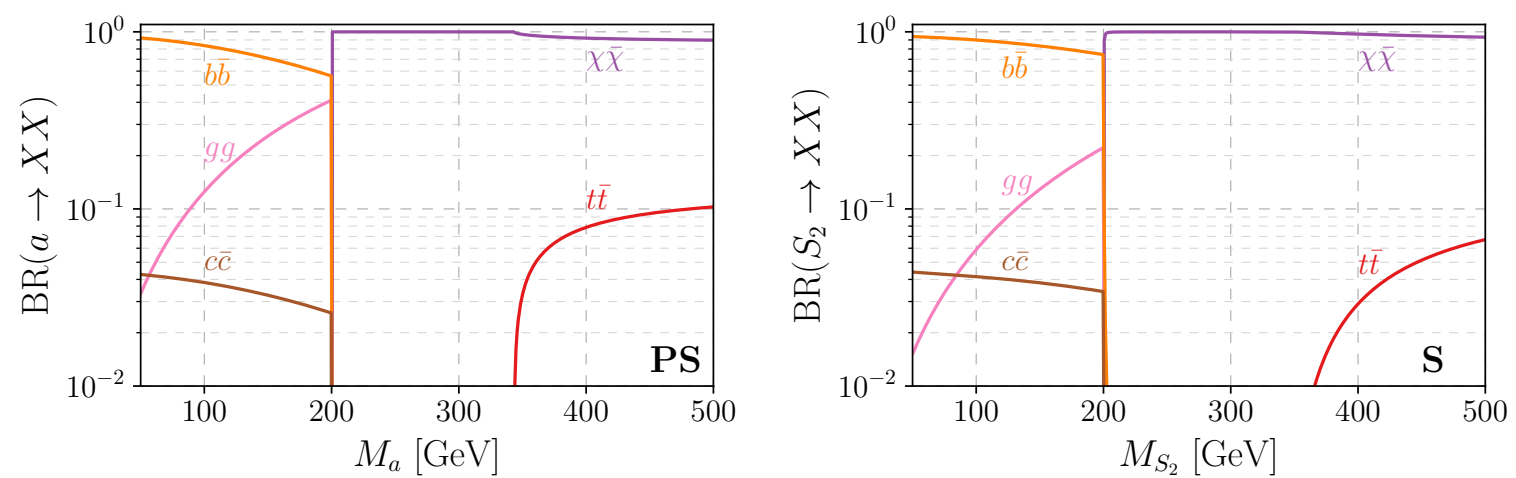

Figure 2. Dominant branching ratios of the light pseudoscalar $a$ in the $2 \mathrm{HDM}+\mathrm{PS}$ (left) and the light scalar $S_{2}$ in the $2 \mathrm{HDM}+\mathrm{S}$ (right) for $\tan \beta=1, m_{\chi}=100 \mathrm{GeV}$ (to show more decay channels), $M_{H / S_{1}}=M_{H^{ \pm}}=M_{A}=500 \mathrm{GeV}$ and other parameter values as given in eq. (4.4).

As mentioned, in the alignment limit the couplings of $h$ to the SM fields substantially coincide with the ones expected for the SM Higgs boson. However, its total width can deviate with respect to the SM prediction, because of the eventual presence of additional decay channels. The most relevant, if kinematically allowed, is the one into a pair of $a$ or $S_{2}$ states, respectively. Even for mediator masses above half of the SM Higgs mass, the three body decay $a \chi \bar{\chi}$, or $S_{2} \chi \bar{\chi}$ respectively, can be sizeable and even dominate for small enough DM masses, see figure 1 (for brevity only the two dominating SM decays are shown).

The width of the additional light (pseudo)scalar shows similar behavior in both models. It is dominated by the $\chi \bar{\chi}$ channel, if kinematically accessible, even if the decay to $t \bar{t}$ is allowed, see figure 2 . The other decay channels arise from mixing with the corresponding doublet state and are therefore suppressed by $\sin ^{2} \theta$.

The decay channels of the heavy scalars $H / S_{1}$, see figure 3 , and pseudoscalars $A$, see figure 4 , are exchanged in the $2 \mathrm{HDM}+\mathrm{PS}$ and $\mathrm{S}$. All four are dominated by the decay to top quarks. In addition the heavy pseudoscalar $A$ in the $2 \mathrm{HDM}+\mathrm{S}$ and the heavy scalar $H$ in the $2 \mathrm{HDM}+\mathrm{PS}$ decay to $Z S_{2}$, or $Z a$ respectively, enabling the resonant production of a mono- $Z$ final state discussed below. It can be seen that, in the $2 \mathrm{HDM}+\mathrm{PS}, \mathrm{BR}(H \rightarrow Z a)$ 

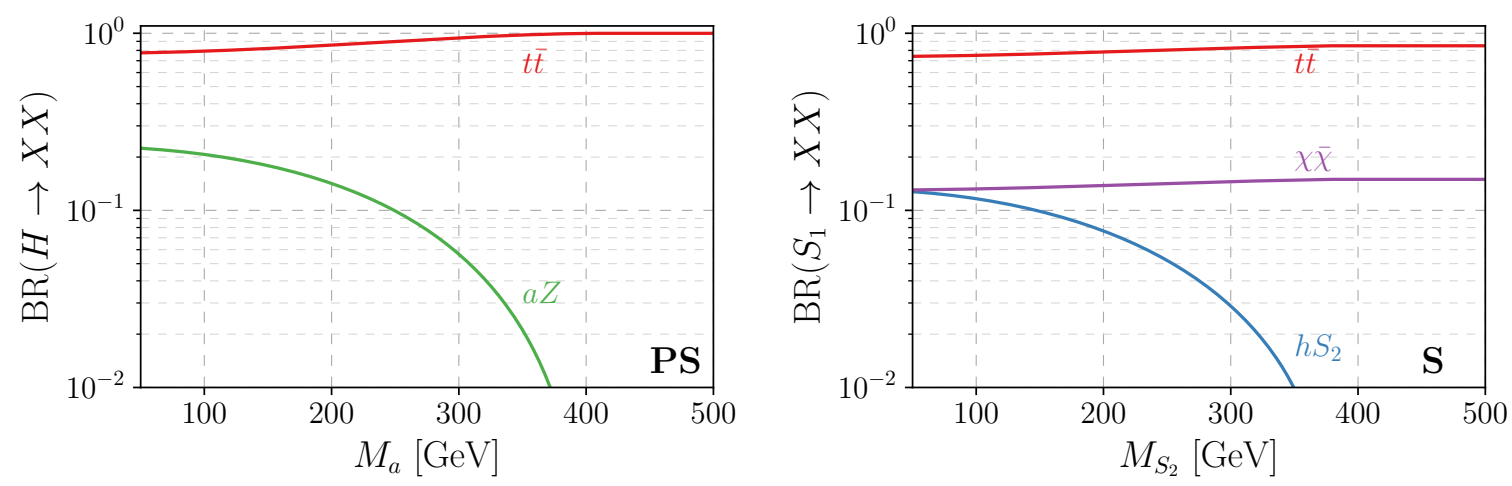

Figure 3. Dominant branching ratios of the heavy scalar $H / S_{1}$ for $\tan \beta=1, M_{H / S_{1}}=M_{H^{ \pm}}=$ $M_{A}=500 \mathrm{GeV}, m_{\chi}=10 \mathrm{GeV}$ and other parameter values as given in eq. (4.4) in the $2 \mathrm{HDM}+\mathrm{PS}$ (left) and $2 \mathrm{HDM}+\mathrm{S}$ (right).
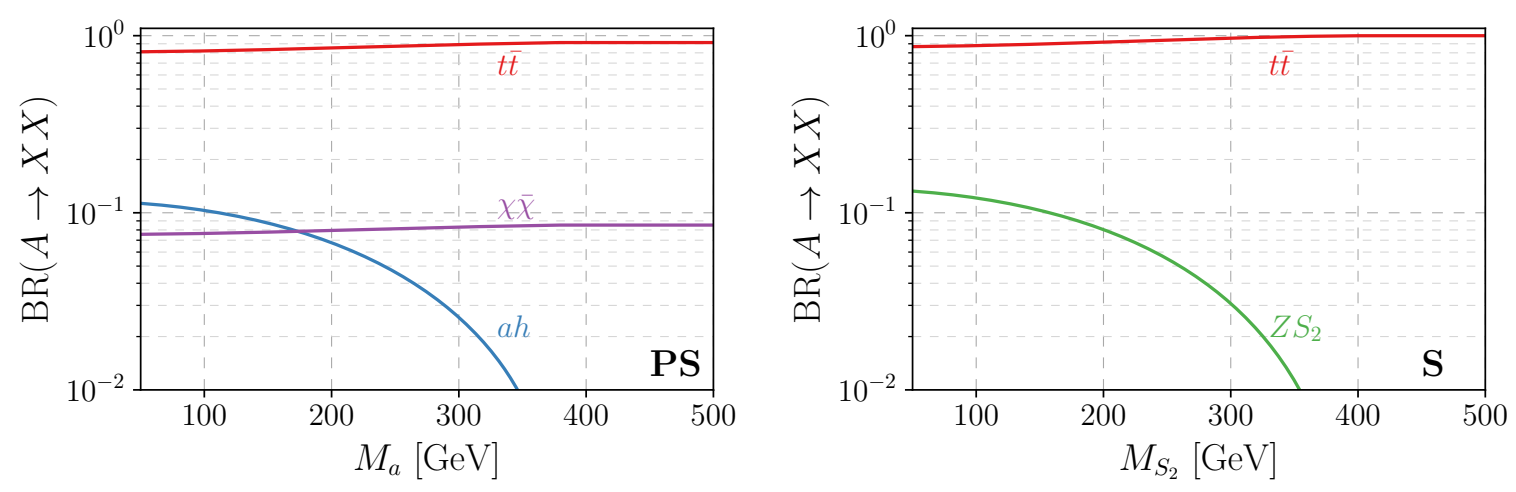

Figure 4. Dominant branching ratios of the heavy pseudoscalar $A$ for $\tan \beta=1, m_{\chi}=10 \mathrm{GeV}$, $M_{H / S_{1}}=M_{H^{ \pm}}=M_{A}=500 \mathrm{GeV}$ and other parameter values as given in eq. (4.4) in the $2 \mathrm{HDM}+\mathrm{PS}$ (left) and $2 \mathrm{HDM}+\mathrm{S}$ (right).

is bigger by roughly a factor of two than $\mathrm{BR}\left(A \rightarrow Z S_{2}\right)$ in the $2 \mathrm{HDM}+\mathrm{S}$. This is due to the smaller decay width of scalars to quarks compared to pseudoscalars, and therefore a smaller total width, resulting in a larger $\mathrm{BR}$ for the $2 \mathrm{HDM}+\mathrm{S}$ than the $2 \mathrm{HDM}+\mathrm{PS}$ to the mono- $Z$ final state. In the $2 \mathrm{HDM}+\mathrm{S}$ the heavy scalar $S_{1}$ has a $\mathrm{BR}$ of $\mathcal{O}(10 \%)$ to $\chi \bar{\chi}$ (from mixing with $S_{2}$ ) and $h S_{2}$, the latter declining with $M_{S_{2}}$. In the 2HDM+PS the analogue is the heavy pseudoscalar $A$, where $\operatorname{BR}(A \rightarrow a h)$ and $\operatorname{BR}(A \rightarrow \chi \bar{\chi})$ are of similar strength. The former decay enables a resonant production of the mono- $h$ final state.

\section{Overview of model constraints}

In this section we give a brief overview on various constraints on the DM properties of the $2 \mathrm{HDM}+\mathrm{S} / \mathrm{PS}$ mainly from non-collider experiments and on $2 \mathrm{HDMs}$ in general as they apply to the studied case and reduce the parameter space. 


\subsection{Direct detection}

Direct detection (DD) phenomenology and their detection prospects are rather different for the two models. In the case of $2 \mathrm{HDM}+\mathrm{S}$ model the DM is coupled to scalar mediators. It consequently generates Spin Independent (SI) interactions due to t-channel exchange of the scalar mediators at tree level, described by the following operator $[7,16]$ :

$$
\mathcal{L}_{N}=c_{N} \mathcal{O}_{1}^{N}=c_{N} \bar{\chi} \chi \bar{N} N
$$

where:

$$
c_{N}=m_{N} \frac{y_{\chi} \sin \theta \cos \theta}{v}\left(\frac{1}{M_{S_{1}}^{2}}-\frac{1}{M_{S_{2}}^{2}}\right)\left(\epsilon_{u} f_{T_{u}}^{N}+\epsilon_{d} \sum_{q=d, s} f_{T_{q}}^{N}+\frac{2}{9} \frac{2 \epsilon_{u}+\epsilon_{d}}{3} f_{T_{g}}^{N}\right)
$$

with the coefficients $\epsilon_{q}$ as given in table 1 and the coefficients $f$ can be found in [34]. Such interaction is well within the reach of current detectors for $y_{\chi} \sim \mathcal{O}(1), \sin \theta \cos \theta \sim \mathcal{O}(0.3)$, $\tan \beta \sim \mathcal{O}(1)$ and $M_{S_{i}} \sim \mathcal{O}(\mathrm{TeV})$, apart from the regions where there are negative interference effects [16]. In the following sections on collider searches we focus on light DM where DD is insensitive, therefore acting as a complementary search.

In the case of the $2 \mathrm{HDM}+\mathrm{PS}$ model, the DM-nucleon interaction, from tree-level exchange of pseudoscalar mediators is described by the following operator [34]:

$$
\mathcal{L}_{N}=\tilde{c}_{N} \mathcal{O}_{4}^{N}=c_{N} \bar{\chi} i \gamma^{5} \chi \bar{N} i \gamma^{5} N
$$

where the effective coefficient $\tilde{c}_{N}$ is given, for example, in [35, 36]. This operator, in the non-relativistic limit, reduces to:

$$
\mathcal{L}_{N} \equiv 4 \tilde{c}_{N}\left(\vec{s}_{\chi} \cdot \vec{q}\right)\left(\vec{s}_{N} \cdot \vec{q}\right)
$$

where $\vec{s}_{\chi}, \vec{s}_{N}$ are the DM and nucleon spins, respectively, while $\vec{q}$ is the momentum transfer. The corresponding cross-section is hence suppressed by the fourth power of the momentum transfer and experimentally relevant only for very light masses of the mediators $[20,35,36]$. Spin independent DM nucleon interactions, within the reach of, especially, next generation detectors like XENONnT and DARWIN, emerge however at the one loop level $[20,21,37-39]$ (see also [19, 40]).

\subsection{Indirect detection and relic density}

Adopting the WIMP paradigm, the DM relic density, as well as eventual Indirect Detection (ID) signals, rely on DM annihilation processes.

The case of the $2 \mathrm{HDM}+\mathrm{S}$ model has been extensively studied e.g. in [7]. The possible annihilation channels include SM fermion-antifermion pairs as well as two scalars and scalar-gauge bosons final states. The full list of annihilation channels is $f \bar{f}, S_{1} S_{1}, S_{1} S_{2}$, $S_{2} S_{2}, H^{+} H^{-}, H^{ \pm} W^{\mp}, A A, A Z, S_{1} h, S_{2} h$, where $f$ denotes all SM fermions with $m_{f}<m_{\chi}$. All these annihilation channels are characterized by p-wave suppressed annihilation crosssections. While it is possible to achieve the correct relic density by suitably accommodating the model parameters, we do not expect signals measurable through ID experiments. 
The phenomenology related to ID and relic density for the $2 \mathrm{HDM}+\mathrm{PS}$ model has been extensively reviewed in [8] (see also [19, 21, 41]). The DM relic density is determined by annihilation processes into $f \bar{f}, X V$, with $X=h, H, H^{ \pm}, a, A$ and $V=W^{ \pm} Z$, and the $a a, a A, A A$ final states. The first two types of annihilation channels have swave dominated cross-sections, hence also relevant for ID (see below). The annihilation cross-sections into pseudoscalars are, instead, p-wave suppressed. The $a a$ channel can be, nevertheless, especially for very light $a$, relevant for the relic density.

Concerning ID, possible signals are mostly accounted by the $b \bar{b}, t \bar{t}$ and $h a$ channels, the latter giving a four fermion, typically $b \bar{b} b \bar{b}$, signature. For heavy DM, the $h A, H Z$ and $H^{ \pm} W^{\mp}$ final states might play a role as well. The $a a, a A$ and $A A$ final states contribute to ID to a negligible extent, because the corresponding cross-sections are p-wave suppressed. As discussed in [8], FERMI-LAT bounds on the $b \bar{b}$ and $t \bar{t}$ channels [42] can probe DM masses from $190 \mathrm{GeV}$ up to around $400 \mathrm{GeV}$, therefore offering a complementary approach to the collider searches. No dedicated studies exist, instead, for annihilation into a gauge and a Higgs boson.

\subsection{Flavour constraints}

As already mentioned, we are considering specific realizations of the $2 \mathrm{HDM}$ in which flavor violation processes are forbidden at tree level through suitable combinations of the couplings of the SM fermions with the different Higgs doublets. FCNC processes can be nevertheless induced at loop level. A comprehensive discussion of the possible bounds has been performed in [43]. Among them, the most relevant ones come from $b \rightarrow s$ transitions, in particular the $B \rightarrow X_{s} \gamma$ process, whose rate is mostly sensitive to $\tan \beta$ and $M_{H^{ \pm}} . b \rightarrow s$ transitions mostly constrain $2 \mathrm{HDM}$ realizations featuring $\tan \beta$ enhanced interactions of the BSM scalars with $d$-type quarks, namely the type II and type Y models. For them we have a lower bound $M_{H^{ \pm}}>570 \mathrm{GeV}$ weakly sensitive to the value of $\tan \beta$. The bound on $M_{H^{ \pm}}$can be translated into bounds for the masses of the other extra scalars in view of the relations imposed by bounds from EWPT and the scalar potential, as illustrated in the subsections below (see also e.g. [22, 41]). Additional constraints, from $B_{s} \rightarrow \mu^{+} \mu^{-}$ and $B \rightarrow K \mu^{+} \mu^{-}$processes, affect the type II model for moderate/high values of $\tan \beta$. Models of type I and type X, having $\tan \beta$ suppressed interactions with the SM quarks, are constrained only in a small region of the parameter space for $\tan \beta \lesssim 2$.

\subsection{Electroweak precision constraints}

It can be shown that the scalar potential for the $2 \mathrm{HDM}+\mathrm{S}$ in eq. (2.1) and (2.18) breaks the custodial symmetry [44-49] (in particular the $\lambda_{4}, \lambda_{5}$ and $\lambda_{h H S}$ terms) and leads to contributions to EW precision observables, unless $M_{A}=M_{H^{+}}$. The most relevant observable is the $\rho$ parameter, which receives a contribution of

$$
\begin{aligned}
\Delta \rho=\frac{1}{(4 \pi)^{2} v^{2}}( & F\left(M_{H^{+}}^{2}, M_{A}^{2}\right)+\cos ^{2} \theta F\left(M_{H^{+}}^{2}, M_{S_{1}}^{2}\right)+\sin ^{2} \theta F\left(M_{H^{+}}^{2}, M_{S_{2}}^{2}\right) \\
& \left.-\cos ^{2} \theta F\left(M_{A}^{2}, M_{S_{1}}^{2}\right)-\sin ^{2} \theta F\left(M_{A}^{2}, M_{S_{2}}^{2}\right)\right),
\end{aligned}
$$


where

$$
F(x, y) \equiv \frac{x+y}{2}-\frac{x y}{x-y} \log (x / y) .
$$

One can verify that $\Delta \rho=0$ for $M_{A}=M_{H^{+}}$. Similarly, for the $2 \mathrm{HDM}+\mathrm{PS}$ potential in eq. (2.7) and (2.12),

$$
\begin{aligned}
\Delta \rho=\frac{1}{(4 \pi)^{2} v^{2}} & \left(F\left(M_{H^{+}}^{2}, M_{H}^{2}\right)+\cos ^{2} \theta F\left(M_{H^{+}}^{2}, M_{A}^{2}\right)+\sin ^{2} \theta F\left(M_{H^{+}}^{2}, M_{a}^{2}\right)\right. \\
& \left.-\cos ^{2} \theta F\left(M_{A}^{2}, M_{H}^{2}\right)-\sin ^{2} \theta F\left(M_{a}^{2}, M_{H}^{2}\right)\right),
\end{aligned}
$$

and $\Delta \rho=0$ for $M_{H}=M_{H^{+}}$. Thanks to our assumption of mass degeneracy, $M_{H}=$ $M_{H^{ \pm}}=M_{A}$ or $M_{A}=M_{H^{ \pm}}=M_{S_{1}}$, we automatically fulfill EW precision constraints. However, as soon as a small mass splitting appears in the spectrum, limits from EW precision can become very constraining if the scalars making up the doublet do not have very similar masses. This implies $M_{S_{1}} \sim M_{A} \sim M_{H^{ \pm}}$and $0 \leq \theta \lesssim \pi / 4$ for the $2 \mathrm{HDM}+\mathrm{S}$ and $M_{A} \sim M_{H} \sim M_{H^{ \pm}}$and $0 \leq \theta \lesssim \pi / 4$ for the $2 \mathrm{HDM}+\mathrm{PS}$. This, together with the fact that to have resonant enhancement of mono- $Z$ and mono- $h$ one of the (pseudo)scalars needs to be below and the other above the top threshold, is the reason that motivates our choice of concentrating on $M_{S_{1}}>M_{S_{2}}$, respectively $M_{A}>M_{a}$. For more details on EW precision constraints for these models, see $[3,7]$.

\subsection{Perturbativity and unitarity constraints}

Perturbativity and unitarity constraints for the $2 \mathrm{HDM}+\mathrm{S}$ model have been studied in detail in [16] (see also $[50,51]$ ). By requiring that the couplings of the scalar potential are perturbative and that the amplitude of scattering processes of the type $\phi_{i} \phi_{j} \rightarrow \phi_{k} \phi_{l}$ involving scalar states preserves unitarity, we obtain the following constraints:

$$
\begin{aligned}
\left|\lambda_{3}\right|+\left|\lambda_{4}\right| & <1 \\
\left|\lambda_{3}\right|+\left|\lambda_{5}\right| & <1 \\
\lambda_{1}+\lambda_{2}+\sqrt{\lambda_{1}^{2}-2 \lambda_{1} \lambda_{2}+\lambda_{2}^{2}+4 \lambda_{5}^{2}} & <2 \\
\lambda_{1}+\lambda_{2}+\sqrt{\lambda_{1}^{2}-2 \lambda_{1} \lambda_{2}+\lambda_{2}^{2}+4 \lambda_{4}^{2}} & <2 \\
\lambda_{11 S}+\lambda_{22 S}+\sqrt{\lambda_{11 S}^{2}-2 \lambda_{11 S} \lambda_{22 S}+\lambda_{22 S}^{2}+4 \lambda_{12 S}^{2}} & <2 .
\end{aligned}
$$

These bounds on the quartic couplings can be also re-expressed into upper limits on the mass splittings between the scalar Higgs eigenstates (see, for example, [16]). Together with eq. (3.8) one should also take into account the following constraints from the requirement that the scalar potential is bounded from below:

$$
\begin{aligned}
\lambda_{1,2, S} & >0 \\
\sqrt{\lambda_{1} \lambda_{2}} & >-\lambda_{3} \\
\sqrt{2 \lambda_{1} \lambda_{S}} & >-\lambda_{11 S} \\
\sqrt{2 \lambda_{2} \lambda_{S}} & >-\lambda_{22 S} \\
\sqrt{\lambda_{1} \lambda_{2}} & >\left|\lambda_{5}\right|-\lambda_{3}-\lambda_{4} .
\end{aligned}
$$


Unitarity constraints for the 2HDM+PS model have been considered, instead, in [18]. These rely on the amplitudes of the processes $a a, a A, A A \rightarrow W W$ and can be expressed through the following condition:

$$
\left|\Lambda_{ \pm}\right|<8 \pi
$$

where

$$
\Lambda_{ \pm}=\left[\frac{\Delta_{H}^{2}}{v^{2}}-\frac{\Delta_{a}^{2}(1-4 \cos 4 \theta)}{8 v^{2}} \pm \sqrt{\frac{\Delta_{H}^{4}}{v^{4}}+\frac{\Delta_{a}^{4}(1-4 \cos 4 \theta)}{8 v^{4}}}\right],
$$

with $\Delta_{a}^{2}=M_{A}^{2}-M_{a}^{2}$ and $\Delta_{H}^{2}=\frac{2 \hat{M}_{12}^{2}}{\sin 2 \beta}-M_{H^{ \pm}}^{2}+M_{W}^{2}-\frac{M_{h}^{2}}{2}$.

Requiring perturbativity on top of unitarity strengthens the limit down to

$$
\left|\Lambda_{ \pm}\right|<\mathcal{O}(1)
$$

\subsection{Collider searches}

In this section, we will focus on the collider searches for $t \bar{t}+\mathbb{E}_{T}$ and mono-jet, whereas $t \bar{t}$, mono- $Z$ and mono- $h$ will be discussed in detail in section 4 , since they lead to the most stringent limits for the $2 \mathrm{HDM}+\mathrm{S} / \mathrm{PS}$.

\subsection{1 $t \bar{t}+\not_{T}$}

New spin-0 mediators with large invisible widths can be searched in the $t \bar{t}+\mathbb{E}_{T}$ (and $\left.b \bar{b}+\mathbb{E}_{T}\right)$ channels. The most recent experimental searches have been reported in $[52,53]$. Their results have been interpreted in the context of the so-called DM-simplified model (DMF). They can be, however, straightforwardly applied to the $2 \mathrm{HDM}+\mathrm{PS}$ model, as long as $M_{a} \ll M_{A}$, by applying the simple scaling relation (a similar relation applies for $b \bar{b}+\mathbb{E}_{T}$ with the replacement of $\tan \beta$ according to table 1)

$$
\frac{\sigma\left(p p \rightarrow t \bar{t}+\mathbb{E}_{T}\right)_{2 \mathrm{HDM}+\mathrm{PS}}}{\sigma\left(p p \rightarrow t \bar{t}+\mathbb{E}_{T}\right)_{\mathrm{DMF}}}=\left(\frac{y_{\chi} \sin \theta}{g_{\chi} g_{q} \tan \beta}\right)^{2} .
$$

As discussed in [8], the limits of $[52,53]$ can be applied, in analogous manner as illustrated above, also to the $2 \mathrm{HDM}+\mathrm{S}$, as long as there is substantial mass splitting between the BSM spin-0 scalars.

In case that the new spin-0 scalars have comparable masses, the $\mathbb{E}_{T}$ spectrum features distortions with respect to the DMF case. More refined procedures to map the experimental limits on the models under study should then be applied. The case of the $2 \mathrm{HDM}+\mathrm{PS}$ model is illustrated in [8].

For the extended 2HDMs we are considering, the $t \bar{t}+\mathbb{E}_{T}$ exclusions are shown to be subdominant [54]. Therefore, we don't derive explicit bounds from those searches.

\subsubsection{Mono-jet}

Similarly to what occurs in heavy flavors + missing energy searches, observational constraints are typically interpreted in the context of simplified models. In the limits in which the 2HDM-like neutral bosons are decoupled, in mass, with respect to the singlet-like states, the kinematic distribution of the mono-jet events are substantially the same for the DMF 
and $2 \mathrm{HDM}+\mathrm{S} / \mathrm{PS}$ models. Experimental limits can be then applied to our setups just by using analogous scaling relations to eq. (3.13).

As mono-jet events provide the strongest bounds among the initial state radiation signatures [55], we explicitly checked promising points with the help of the CheckMATE [56] implementation of the latest ATLAS search [4] and found no excluded points for $\tan \beta=1$. As shown in [3], mono-jet limits only arise for $\tan \beta<1$, however, this region is already excluded by other constraints and therefore we do not investigate this further.

\section{Comparison of 2HDM + scalar/pseudoscalar and their LHC signatures}

After the general overview of constraints in the last section, we will now turn to the $2 \mathrm{HDM}+\mathrm{S} / \mathrm{PS}$ specific LHC signatures and limits we obtained for the leftover parameter space by dedicated collider simulations.

\subsection{General aspects}

Before describing the simulations and discussing our parameter space in detail, let us first have a brief look at resonant production processes, which can be described analytically and will become handy for the interpretation of the simulation results.

Resonant production. The production cross section for a spin-0 scalar (or pseudoscalar) resonance $S$, with mass $M$ and total decay width $\Gamma_{\text {tot }}$, subsequently decay into the final state $X$, can be schematically written, in the narrow width approximation, as [57]

$$
\sigma(p p \rightarrow S \rightarrow X)=\frac{\Gamma(S \rightarrow X)}{M \Gamma_{\mathrm{tot}} s} \sum_{i} C_{i} \Gamma(S \rightarrow i)=\frac{1}{M s} \operatorname{BR}(S \rightarrow X) \sum_{i} C_{i} \Gamma(S \rightarrow i) .
$$

The sum over $i$ runs over the possible partonic initial states, for example quark or gluon pairs, $C_{i}$ are weight factors that account for the protons PDFs and colour factors, and $s=(13 \mathrm{TeV})^{2}$ is the center of mass energy squared. The values of the $C_{i}$ are given by:

$$
\begin{aligned}
C_{g g} & =\frac{\pi^{2}}{8} \int_{M^{2} / s}^{1} \frac{\mathrm{d} x}{x} g(x) g\left(\frac{M^{2}}{s x}\right), \\
C_{q \bar{q}} & =\frac{4 \pi^{2}}{9} \int_{M^{2} / s}^{1} \frac{\mathrm{d} x}{x}\left[q(x) \bar{q}\left(\frac{M^{2}}{s x}\right)+q\left(\frac{M^{2}}{s x}\right) \bar{q}(x)\right],
\end{aligned}
$$

with $q(x)(\bar{q}(x))$ and $g(x)$ being, respectively, the parton distribution functions (PDFs) of quarks (anti-quarks) and gluons inside the proton and $x$ is the conventional Bjorken scaling variable.

In general, the two dominant production mechanisms are gluon fusion and $b \bar{b}$ initial states. We show the production cross sections of the heavy (pseudo)scalar, as a function of the resonance mass for both production modes in figure 5 . The left panel shows the production cross section for gluon fusion, the right panel for $b \bar{b}$ initial states. For $\tan \beta=1$, the production cross section from gluon fusion results to be about 100 times larger than from $b \bar{b}$ initial states. This dominance of gluon fusion production depends on the 2HDM Yukawa type in combination with the value of $\tan \beta$, but holds for all 2HDM Yukawa types 

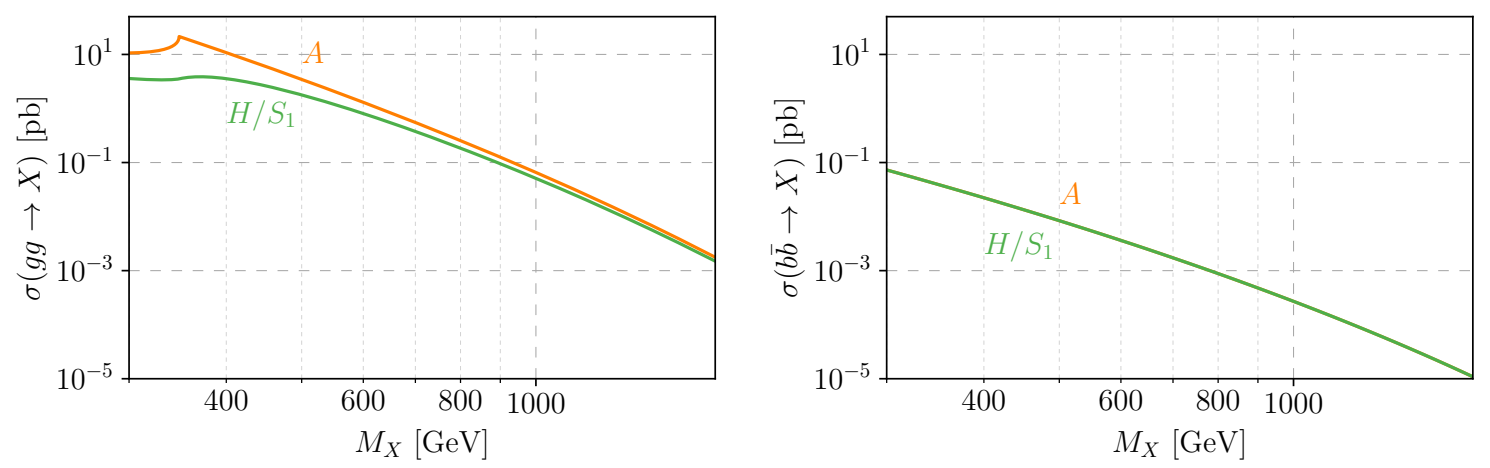

Figure 5. Production cross sections for the heavy scalar $H / S_{1}$ and pseudoscalar $A$ through gluon fusion (left) and $b \bar{b}$ initial state (right), in the $2 \mathrm{HDM}+\mathrm{PS} / \mathrm{S}$ as given in eq. (4.1). The plot depicts the case of $\tan \beta=1$.

in the whole parameter space we take into consideration $(0.3 \leq \tan \beta \leq 3)$, so in our simulations we can safely neglect the $b \bar{b}$ production mode.

From figure 5, we can also see that the production of a pseudoscalar particle always results in a larger cross section. This is because the effective coupling of the pseudoscalar mediator is larger, from same assignation of the Yukawa couplings, with respect to the scalar mediator, see e.g. [41, 58].

Parameter overview. For a better overview we briefly summarize our choice of parameter values, which are motivated by various constraints discussed above and used for the following Monte Carlo simulations and other examples throughout the paper:

$$
\begin{aligned}
M_{A} & =M_{H^{ \pm}}=M_{H / S_{1}} \\
\lambda_{3} & =M_{h}^{2} / v^{2} \\
\lambda_{i i S / P} & =0 \\
y_{\chi}^{S, P S} & =1 \\
m_{\chi} & =10 \mathrm{GeV} \\
\sin \theta & =0.3 \\
\tan \beta & \in[0.3,3] .
\end{aligned}
$$

Signal generation. We simulate events for the signal processes with MadGraph5 aMC@NLO [59-63] at next-to-leading order (NLO) in QCD using the 263000 PDF set (NNPDF3.0) [64] provided through LHAPDF6 [65]. For the parton-showering we use the MadGraph built-in Pythia 8.2 [66]. A fast detector simulation is done with Delphes 3.4.2 [67] using the provided CMS detector card. The final cuts are implemented in MadAnalysis 5 [68, 69].

The correct implementation of the program chain and analysis is checked by reproducing the mono- $Z$ and mono- $h$ exclusions for the $2 \mathrm{HDM}+\mathrm{PS}$ presented in $[8,54]$.

In the following, we will look at the different channels that provide the strongest limits for the $2 \mathrm{HDM}+\mathrm{S} / \mathrm{PS}$ model. 


\section{$4.2 t \bar{t}$ resonances}

For masses above the top-threshold, $M_{H / S_{1}, A}>2 m_{t}$, the additional heavy Higgses dominantly decay into a pair of top quarks, cf. section 2.4. Searches for $t \bar{t}$ resonances are therefore a powerful tool to test $2 \mathrm{HDMs}$ in general and $2 \mathrm{HDM}+\mathrm{S} / \mathrm{PS}$ in particular.

One aspect that complicates the analysis, is that the signal processes interfere nontrivially with the SM background, as pointed out and taken into account by the experimental analysis in [70]. There the ATLAS results are interpreted in a $2 \mathrm{HDM}$ of type II, for the case in which the individual contribution of the two mediators to the signal can be singled out as well as for the mass-degenerate case where both mediators contribute. As the later case gives significantly stronger constraints, deriving exclusions for a single mediator serves as a conservative estimate.

The most recent bounds for $\sqrt{s}=13 \mathrm{TeV}$ and an integrated luminosity of $35.9 \mathrm{fb}^{-1}$ are provided by CMS in [71]. The limits are presented in terms of simplified models with either a scalar or a pseudoscalar and Yukawa-like couplings to tops and in the hMSSM. Here, a $1.9 \sigma$ "signal-like deviation" is observed that would fit to a pseudoscalar with a mass of around $400 \mathrm{GeV}$, therefore the limits in that mass range are not significantly stronger than the ones from the previous ATLAS analysis with $\sqrt{s}=8 \mathrm{TeV}$.

The additional light state $a / S_{2}$ can have a non-trivial impact on the limit due to interference effects, cf. section 7.1 in [8]. However, this effect is expected to be small for our choice of small mixing $\sin \theta=0.3$. A detailed analysis of the impact of interference and combining the limits for the two heavy states is beyond the scope of this article. Instead, we recast the recent CMS limits [71] for single mediators to our parameter space, interpolating between the different total width to mass ratios given in the paper. As commented above in the context of [70], this can bee seen as a conservative estimate, since the limits get significantly stronger by taking the contribution of both mass-degenerate states $H / S_{1}$ and $A$ into account.

The limits are shown in figure 6 in the $M_{A, H / S_{1}}-\tan \beta$-plane for the $2 \mathrm{HDM}+\mathrm{S}$ (top) and $2 \mathrm{HDM}+\mathrm{PS}$ (bottom). We choose the setting in eq. (4.4) and $M_{a / S_{2}}=400 \mathrm{GeV}$. There exists a mild dependency on the mass of the light state due to changes of the total width which is shown in figure 13 and 14 .

The decay widths of pseudoscalars to quarks and gluons (for $M_{A}$ above the top threshold) are bigger than the ones of scalars, cf. section 2.4, appendix A and [72]. Together with the effective coupling approximation for the dominant gluon fusion production, cf. eq. (4.1) and [73], it can be understood that pseudoscalar resonances are expected to provide a stronger constraint than scalar ones. In the $2 \mathrm{HDM}+\mathrm{S}$ the couplings of the heavy scalar $S_{1}$ are reduced due to the mixing with the singlet $S_{2}$, while the pseudoscalar couplings are untouched. As a consequence the exclusion for $S_{1}$ reaches up to $\tan \beta=1$, while the one for $A$ clearly exceeds $\tan \beta=1$. In the $2 \mathrm{HDM}+\mathrm{PS}$ instead, the stronger constrained pseudoscalar mixes with the singlet $a$, weakening the exclusion and leading to overall weaker constraints from $t \bar{t}$ searches for this model.

In both models, for masses of the heavy Higgs around $500 \mathrm{GeV}, t \bar{t}$ resonance searches provide a strong lower limit on $\tan \beta$ and essentially exclude values below $\tan \beta \lesssim 1$. 

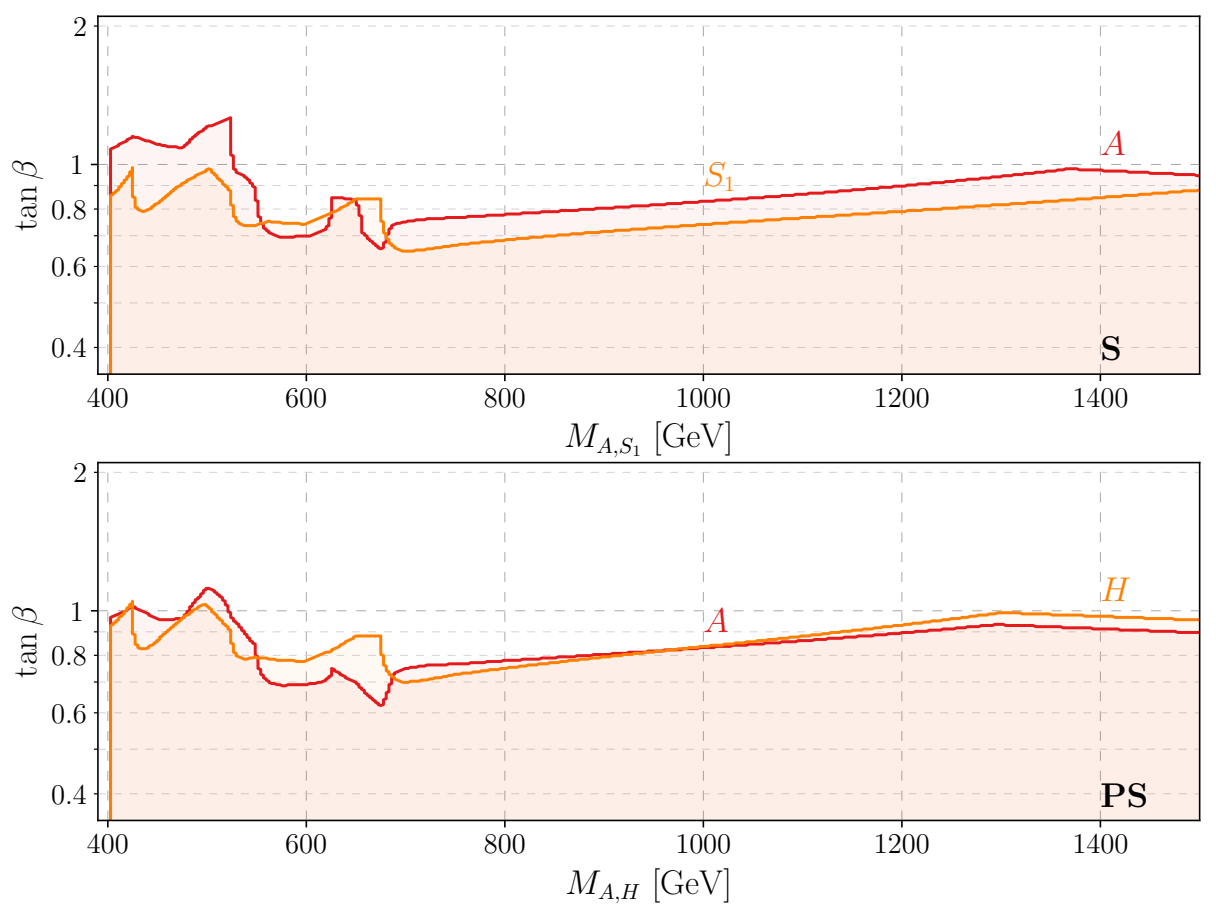

Figure 6. $2 \sigma$ observed exclusion limits from $t \bar{t}$ resonance searches by CMS [53] in the $M_{A, H / S_{1}}-$ $\tan \beta$-plane for the $2 \mathrm{HDM}+\mathrm{S}$ (top) and $2 \mathrm{HDM}+\mathrm{PS}$ (bottom). The parameters are fixed to the values from eq. (4.4) and $M_{a / S_{2}}=400 \mathrm{GeV}$. The limits derived from searches for a scalar (pseudoscalar) resonance $H / S_{1}(A)$ are given in orange (red).
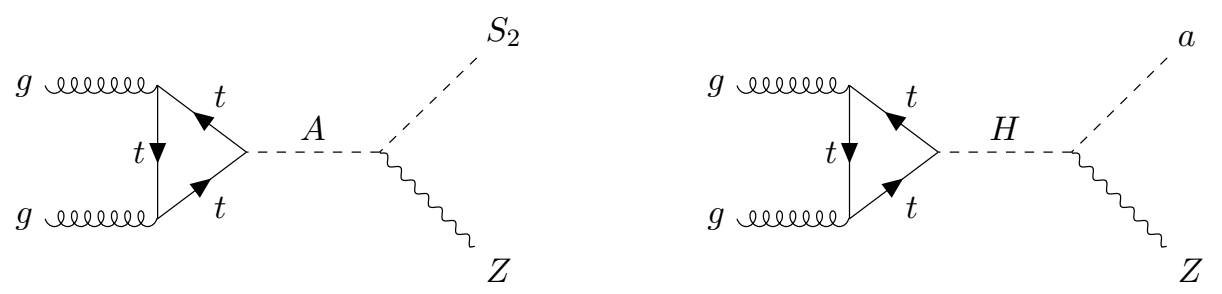

Figure 7. Feynman diagrams for the resonant production of a mono- $Z$ signal via gluon fusion production in the chosen mass hierarchy for the $2 \mathrm{HDM}+\mathrm{S}$ (left) and $2 \mathrm{HDM}+\mathrm{PS}$ (right).

\subsection{Mono- $Z$}

For a strong mono- $Z$ signal, which is a general feature for 2 HDMs extended by pseudoscalar mediated DM [74], the heavy neutral spin-0 particle of the doublet which doesn't mix with the singlet has to be produced, meaning the scalar $H$ in the $2 \mathrm{HDM}+\mathrm{PS}$ and the pseudoscalar $A$ in the $2 \mathrm{HDM}+\mathrm{S}$. This resonantly produced state can decay to a $Z$ boson and the light state $a$ or $S_{2}$, respectively, which further decay to $\chi \bar{\chi}$ with a high branching ratio, cf. section 2.4. The Feynman diagrams for the processes are shown in figure 7 for the dominant gluon fusion production.

Searches for the relatively clean final state, where the $Z$ boson decays leptonically (meaning to electrons or muons), in association with $\mathbb{E}_{T}$ are performed by the ATLAS and CMS collaborations [75, 76]. 
$\boldsymbol{E}_{\boldsymbol{T}}$ spectrum. The maximum value of the missing transverse energy $\mathbb{E}_{T}$ can be obtained from kinematics and is given by [3]

$$
\mathbb{E}_{T}^{\max }=\frac{\lambda^{1 / 2}\left(M_{A, H}, M_{S_{2}, a}, M_{Z}\right)}{2 M_{A, H}},
$$

where $\lambda\left(m_{1}, m_{2}, m_{3}\right)$ is given in eq. (A.17) and the first (second) subscript is used for the $2 \mathrm{HDM}+\mathrm{S}(\mathrm{PS})$. The missing energy spectrum is given by

$$
\frac{1}{N} \frac{d N}{d \mathbb{E}_{T}}=\frac{\mathbb{E}_{T}}{2 \mathbb{E}_{T}^{\max } \sqrt{\left(\mathbb{E}_{T}^{\mathrm{max}}\right)^{2}-\mathbb{E}_{T}^{2}}},
$$

that is a monotonic increasing function in $\mathscr{E}_{T}$. However, detector smearing effects are expected to increase the maximum value of $\mathbb{E}_{T}$ to $\mathbb{E}_{T}^{\max , D}>\mathbb{E}_{T}^{\max }$ and one expects the resulting distribution to be peaked (instead of having its endpoint) close to $\mathbb{E}_{T}=\mathbb{E}_{T}^{\max }$.

Two example $\mathbb{E}_{T}$ spectra after detector simulation for the $2 \mathrm{HDM}+\mathrm{PS}$ in the $e^{+} e^{-}+\mathbb{E}_{T}$ final state are shown in figure 8 together with the predicted SM backgrounds and the observed number of events as provided by ATLAS [75]. Nearly identical $\mathbb{E}_{T}$ spectra exist for the $\mu^{+} \mu^{-}+\mathbb{E}_{T}$ final state. As described below both final states are combined to determine the exclusion limits. The signal features a peak a bit below $\mathbb{E}_{T}=M_{A} / 2$ on top of the smoothly falling SM background.

Backgrounds. For mono- $Z$ searches the main irreducible background is $Z Z$ production with one $Z$ decaying to neutrinos. Another important background is $W Z$ production, where one lepton from the $W$-decay escapes detection or a $\tau$ decays hadronically, cf. figure 8 . Minor backgrounds are $Z+$ jets processes with poor $\mathbb{E}_{T}$ reconstruction and non-resonant $\ell \ell$ production. In [75], the backgrounds are estimated from simulations and data-driven methods. For both the electron and muon final state the background uncertainty is dominated by systematic errors which are driven by the uncertainty on the $Z+$ jets background.

Current constraints. To determine model constraints we use the ATLAS results [75] as they are also used by the LHC-DMWG and easier to reproduce than the (slightly stronger) CMS results [76]. To be explicit, for our exclusion bounds, we use the expected number of background events $b$ and the corresponding uncertainty $\sigma_{b}$ from [75], together with our simulated event numbers $s$ for various parameter points and the sensitivity formula from $[8,77]$ :

$$
Z_{i}=\sqrt{2\left((s+b) \ln \left[\frac{(s+b)\left(b+\sigma_{b}^{2}\right)}{b^{2}+(s+b) \sigma_{b}^{2}}\right]-\frac{b^{2}}{\sigma_{b}^{2}} \ln \left[1+\frac{\sigma_{b}^{2} s}{b\left(b+\sigma_{b}^{2}\right)}\right]\right)} .
$$

The index $i$ refers to the different bins and the values for $Z_{i}$ are added up quadratically to find the (square of the) overall sensitivity $Z^{2}$, where one expects to exclude parameter points with $Z>2$ at $95 \%$ confidence level. In this way we obtain the expected exclusion limits, however, as they are very similar to the observed limits, cf. [54], we can use the expected mono- $Z$ limits to compare them to the observed $t \bar{t}$ and mono- $h$ ones in section 4.5. 


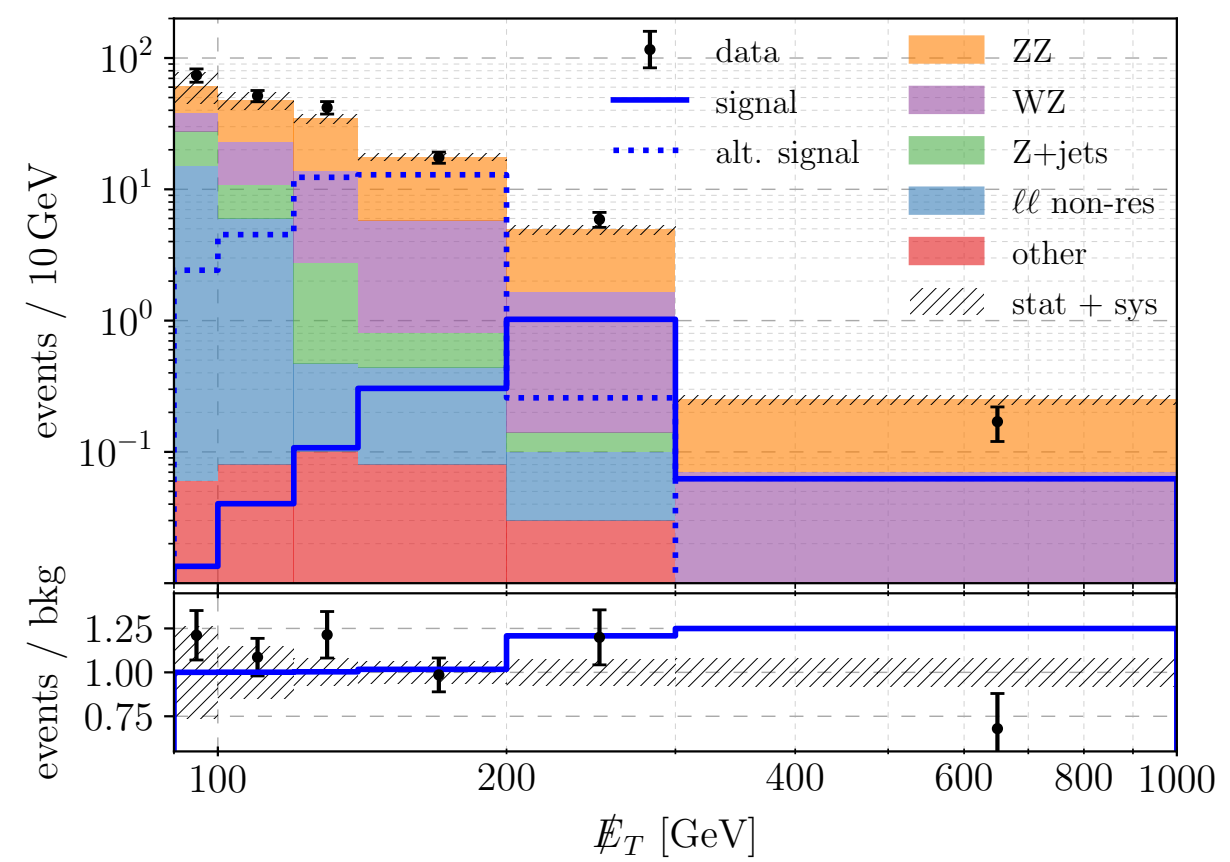

Figure 8. Top panel: $\mathbb{E}_{T}$ spectra for $g g \rightarrow e^{+} e^{-} \chi \bar{\chi}$ in the $2 \mathrm{HDM}+\mathrm{PS}$ for $M_{a}=250 \mathrm{GeV}$, $M_{A}=700 \mathrm{GeV}$ (solid line) and $M_{a}=150 \mathrm{GeV}, M_{A}=400 \mathrm{GeV}$ (dotted line), while the other parameters are set to the values given in eq. (4.4). The expected SM backgrounds and observed events are taken from [75] and shown in different colors with their combined uncertainty displayed as a hatched region on top. Lower panel: ratios of the observed events (points with error-bars) and signal + background expectation (solid line) to the background expectation plotted together with the background uncertainty (hatched region).

The constraints from mono- $Z$ searches are similar in shape and reach for both models, see figure 9. This can be understood with help of the approximation in eq. (4.1) and its corresponding discussion: on the one hand, in the $2 \mathrm{HDM}+\mathrm{S}$ a heavy pseudoscalar $A$ needs to be produced, which happens with a production cross section that is bigger by roughly a factor of two than the one for the production of the scalar $H$ in the 2HDM+PS, cf. figure 7 for the Feynman diagrams. On the other hand, the relevant branching ratios for the mono- $Z$ final state

$$
\mathrm{BR}\left(A \rightarrow S_{2} Z\right) \approx \mathrm{BR}(H \rightarrow a Z) / 2 \approx 0.1,
$$

is bigger in the $2 \mathrm{HDM}+\mathrm{PS}$ by a factor of approximately two, compensating for the smaller production cross section. The difference in the branching ratios is related to the different decay width of scalars and pseudoscalars into top quarks, as mentioned in section 2.4.

The general features of the constraints for the mono- $Z$ channel depicted in figure 9 can be readily understood by considering kinematics and the couplings. In the $M_{a / S_{2}}-\tan \beta$ plot, the weakening of the exclusion limit for larger $\tan \beta$ values is due to the fact that the top-coupling scales like $(\tan \beta)^{-1}$ and the top-coupling is essential for the production of the intermediate heavy Higgs, cf. figure 7. For the $M_{a / S_{2}}-M_{A}$-plot, there are three different features that can be understood: first, the "diagonal" lower bound to the exclusion region 

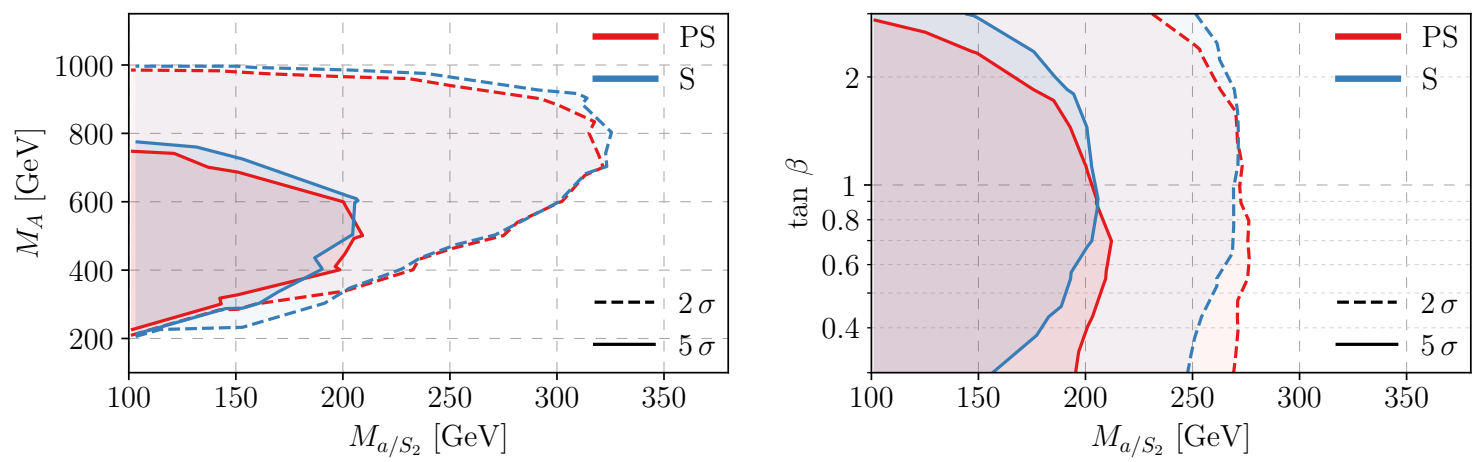

Figure 9. $2 \sigma$ (dashed) and $5 \sigma$ (solid) exclusion limits from mono- $Z$ searches in the $M_{a / S_{2}}-M_{A^{-}}$ plane (left) and $M_{a / S_{2}}-\tan \beta$-plane (right) for the $2 \mathrm{HDM}+\mathrm{S}$ (blue) and PS (red). In both plots we use the parameters as given in eq. (4.4) with $\tan \beta=1$ for the left one and $M_{A}=500 \mathrm{GeV}$ for the right one.

is due to the fact that for $M_{A} \lesssim M_{a / S_{2}}+M_{Z}$, resonant production is not allowed with on-shell $a / S_{2}$. Second, the upper bound of the exclusion limit stems from the heavy Higgs being the harder to produce the heavier it is and third, a heavier $M_{a / S_{2}}$ leaves less energy available for the $Z$, so the $Z$ production gets kinematically suppressed, thereby smoothing out the transition between the first two features.

\subsubsection{Projected sensitivity}

With the detailed data of the experimental analysis at hand [75], we estimate the reach of the high luminosity phase of the LHC (HL-LHC). To do so, we assume integrated luminosities of $300 \mathrm{fb}^{-1}$ and $3000 \mathrm{fb}^{-1}$ and took a projected reduction of systematic uncertainties by $50 \%$ into account called YR18 scenario [78]. The projections for the pseudoscalar model are shown in figure 10 and are nearly identical to the ones for the scalar model, as can be seen for the current bounds in figure 9. From figure 10, one can see that the increased integrated luminosity will lead to a substantial increase in sensitivity, strengthening the $5 \sigma$ limits by roughly a factor of two in terms of the masses for $3000 \mathrm{fb}^{-1}$. In contrast to that, the influence of the improved systematic uncertainties will likely be small.

\subsection{Mono- $h$}

The most recent searches for mono- $h$ with $h \rightarrow b \bar{b}$ by ATLAS and CMS can be found in $[79,80]$. Furthermore, in [81] CMS performs a first search of mono- $h$ with $h \rightarrow b \bar{b}$, $\gamma \gamma, \tau^{+} \tau^{-}, W^{+} W^{-}, Z Z$, where the exclusions are dominated by the $b \bar{b}$ channel. For our analysis, we use the model-independent upper limits on the $h+\mathbb{E}_{T}$ cross section provided by ATLAS [79] and compare it to the one we find from our simulation, as also done by the LHC-DMWG.

Backgrounds. The main backgrounds for mono- $h$ with $h \rightarrow b \bar{b}$ are $t \bar{t}+$ single top as well as leptonically decaying vector bosons + jets processes [79]. In case of $t \bar{t}+$ single top, a top decaying to a bottom quark can in look like it came from a Higgs decay combination with another b-tagged jet, while the missing energy is in neutrinos. Similarly, decaying 

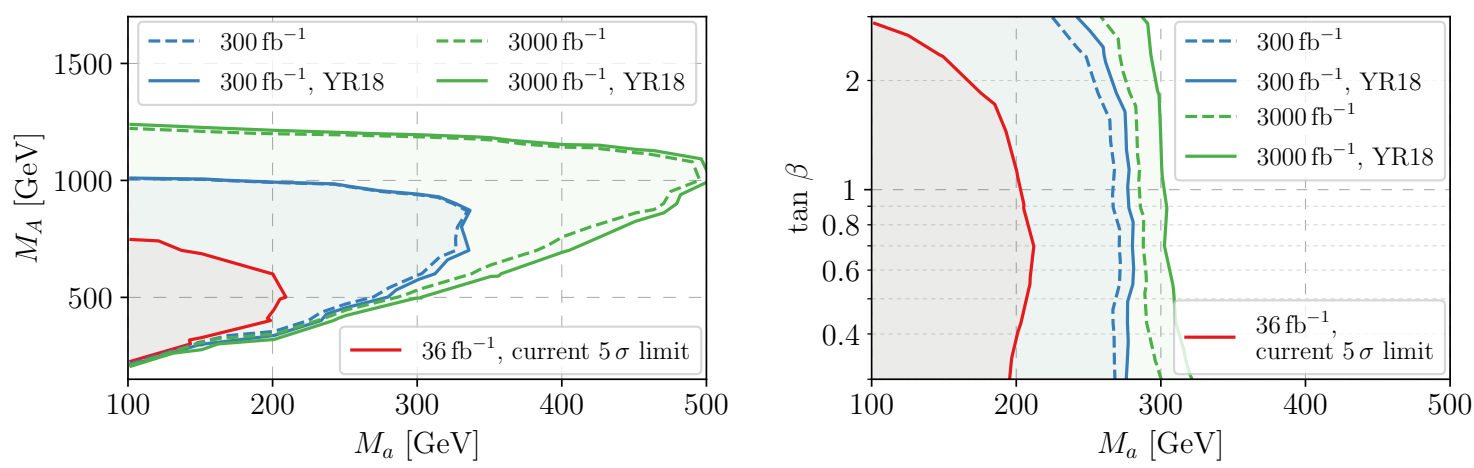

Figure 10. Current $5 \sigma$ exclusion limits for the $2 \mathrm{HDM}+\mathrm{PS}$ (in red) and projected ones for the high-luminosity LHC (in blue and green) for mono- $Z$ searches in the in the $M_{a}-M_{A}$-plane (left) and $M_{a}-\tan \beta$-plane (right). The $2 \mathrm{HDM}+\mathrm{S}$ limits are nearly identical and therefore not shown. The dashed lines correspond to a scenario without any improvement in the systematic uncertainties, whereas the solid lines assume a reduction by $50 \%$, called YR18 scenario. For the other parameters, the numerical values used are identical to figure 9 .

vector bosons also produce $\mathbb{E}_{T}$ via decays to neutrinos or missed charged leptons and the jets can by chance look like a Higgs decay. To generate those backgrounds Monte-Carlo simulations are used in [79].

Current constraints. The non-deviation of the observed events from the SM background is translated into (model-independent) upper limits on the $h(b \bar{b})+\mathbb{E}_{T}$ cross section, which we use in the following by comparing our simulated cross-section to this upper limit, as also done by [8]. In [79], only one $\mathbb{E}_{T}$ bin is used at a time to minimize the model dependency, which implies that the derived limits are conservative estimates. Furthermore, the dependency of the limit on the kinematics within one bin and on the acceptance and efficiency is calculated for several parameter points of a benchmark model and the least stringent limits are given - again, this leads to conservative estimates for the exclusion limits of the $2 \mathrm{HDM}+\mathrm{S} / \mathrm{PS}$, which are shown in figure 11.

Similarly to the mono- $Z$ exclusion plots (cf. figure 9 ), we can see that for larger $\tan \beta$, $M_{A}$ and $M_{a / S_{2}}$ the exclusion limits in each case weaken, as they also do in the case of $M_{a / S_{2}} \sim M_{A}$.

The otherwise most prominent feature in the $M_{a / S_{2}}-M_{A}$-plane (cf. left panel of figure 11), is the dip in the exclusion limits around $M_{A} \sim 700 \mathrm{GeV}$. It originates from a binning effect due to the large $\mathbb{E}_{T}$-bins used in the ATLAS analysis. For $M_{A}>700 \mathrm{GeV}$ a significantly higher fraction of the signal events reaches $\mathbb{E}_{T}>350 \mathrm{GeV}$ (as can be seen from the simulated data) and therefore ending up in the stronger constrained bin reaching from $\mathbb{E}_{T}=350$ to $500 \mathrm{GeV}$. This (over-)compensates the decrease in production cross section by making the mediator heavier, which is the dominant effect between $M_{A}=600$ and $M_{A}=700 \mathrm{GeV}$, leading to a comparably strong or even stronger limit. The effect of more events ending up in the bin of $\mathbb{E}_{T}=350$ to $500 \mathrm{GeV}$ can also be understood in the light of the $\mathbb{E}_{T}$ spectrum discussion in case of the mono- $Z$ (cf. section 4.3 and figure 8). Since the $\mathbb{E}_{T}$ spectrum does not qualitatively change by replacing the $Z$ with an $h$, for 

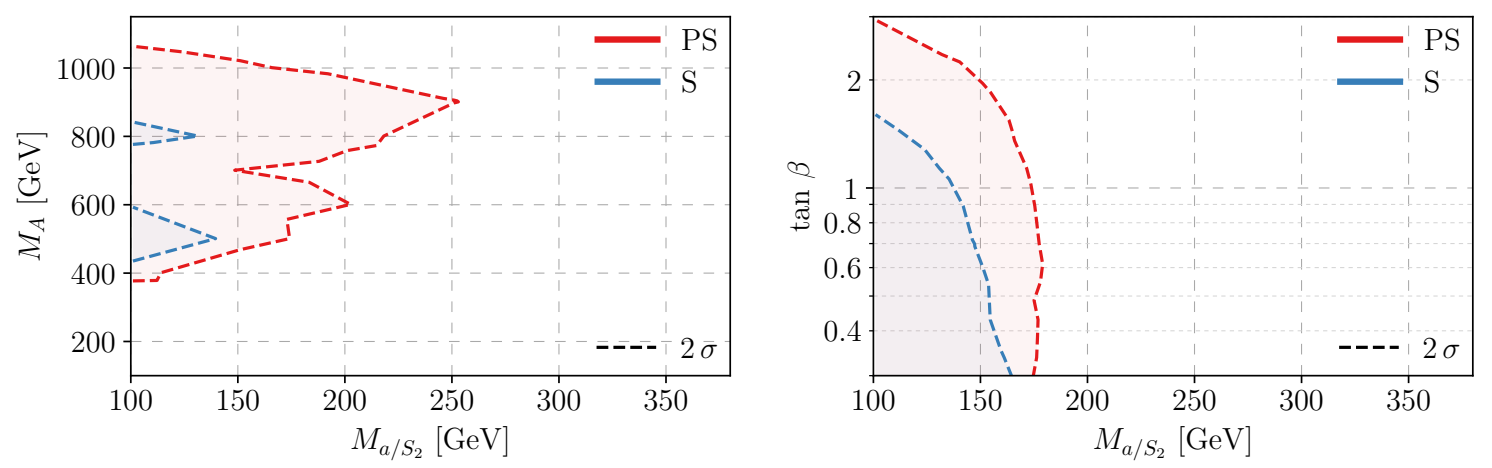

Figure 11. $2 \sigma$ exclusion limits from mono- $h$ searches in $M_{a / S_{2}}-M_{A}$-plane (left) and $M_{a / S_{2}}-\tan \beta$ plane (right) for the $2 \mathrm{HDM}+\mathrm{S}$ (blue) and PS (red). In both plots we use the parameters as given in eq. (4.4) with $\tan \beta=1$ for the left one and $M_{A}=500 \mathrm{GeV}$ for the right one.
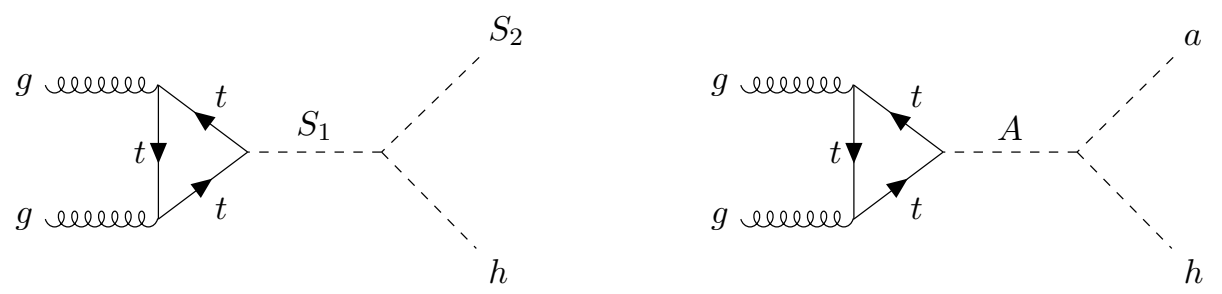

Figure 12. Feynman diagrams for the resonant production of a mono- $h$ signal via gluon fusion production in the chosen mass hierarchy for the $2 \mathrm{HDM}+\mathrm{S}$ (left) and $2 \mathrm{HDM}+\mathrm{PS}$ (right).

values of $M_{A}>700 \mathrm{GeV}$ the peak of the $\mathbb{E}_{T}$ spectrum starts to shift from the $\mathbb{E}_{T}=200$ to $350 \mathrm{GeV}$ bin to the $\mathbb{E}_{T}=350$ to $500 \mathrm{GeV}$ bin because the peak is located a bit below $\mathbb{E}_{T}=M_{A} / 2$.

In contrast to the mono- $Z$ searches, the exclusions limits from mono- $h$ differ significantly between the $2 \mathrm{HDM}+\mathrm{S}$ and $2 \mathrm{HDM}+\mathrm{PS}$. The upper bound on the mass of the light pseudoscalar $a$ is stronger by approximately a factor two in the $2 \mathrm{HDM}+\mathrm{PS}$ than the one for the corresponding scalar $S_{2}$ in the $2 \mathrm{HDM}+\mathrm{S}$. This is due to the fact that, to resonantly produce mono- $h$ events in the $2 \mathrm{HDM}+\mathrm{S}$, one needs to produce the heavy scalar $S_{1}$, rather than the heavy pseudoscalar $A$, and the production cross section for scalars is smaller than the one for pseudoscalars, cf. eq. (4.1) and figure 5. The opposite happens for the $2 \mathrm{HDM}+\mathrm{PS}$, where again the roles of the heavy scalar and pseudoscalar are switched going from mono- $Z$ to mono- $h$, see also the corresponding Feynmann diagrams in figure 7 and 12 . Opposite to the mono- $Z$ case, the branching ratios relevant for mono- $h$ events are similar in both models, cf. section 2.4:

$$
\operatorname{BR}\left(S_{1} \rightarrow S_{2} h\right) \approx \operatorname{BR}(A \rightarrow a h) \approx 0.1
$$

Therefore the $2 \mathrm{HDM}+\mathrm{PS}$ is expected to have a higher mono- $h$ cross section in the resonant region and as the kinematics do not change significantly, this results in stronger exclusion bounds. 

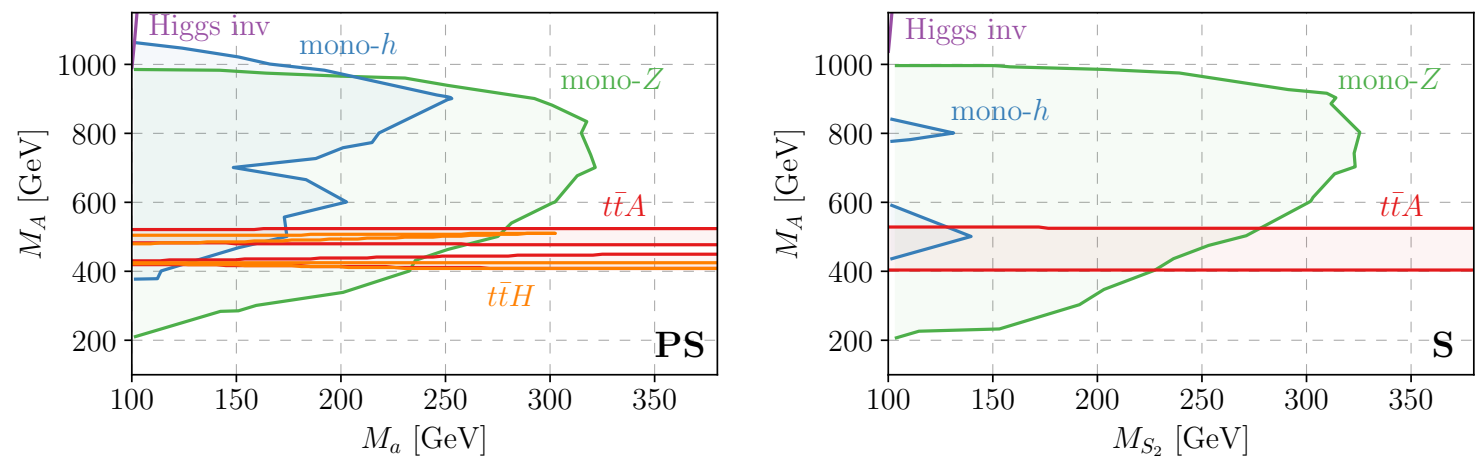

Figure 13. Overview of the $2 \sigma$ exclusion limits from mono- $h$, mono- $Z, t \bar{t}$ resonance (for scalar and psedoscalar mediator) and Higgs to invisible searches (see text for details) in the $M_{a / S_{2}}-M_{A}$-plane for the $2 \mathrm{HDM}+\mathrm{PS}$ (left) and $2 \mathrm{HDM}+\mathrm{S}$ (right). In both plots we use the parameters as given in eq. (4.4) and $\tan \beta=1$.
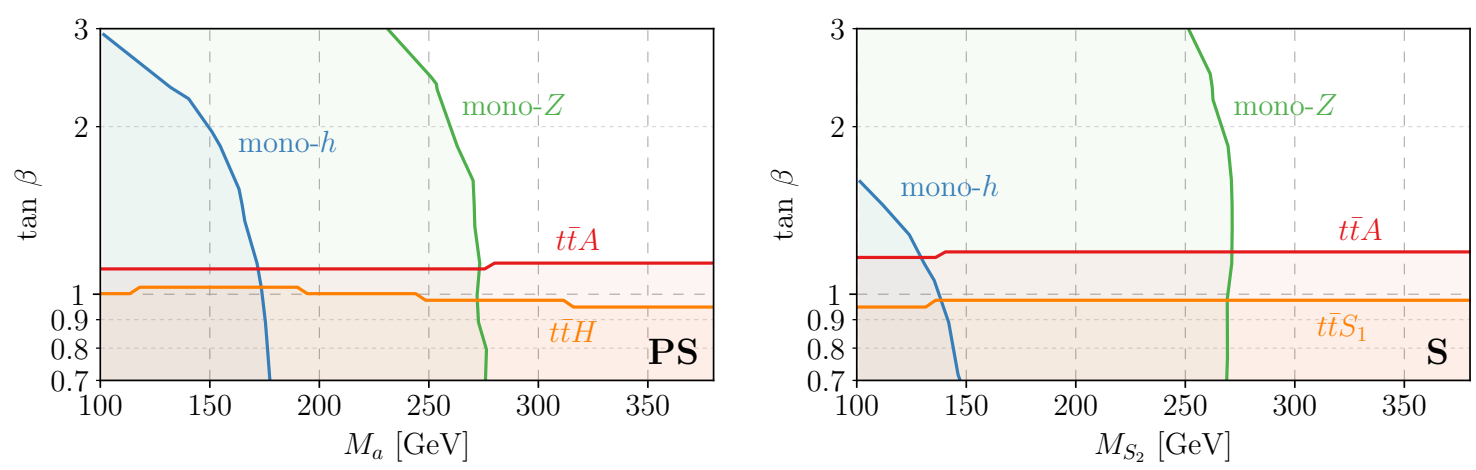

Figure 14. Overview on the $2 \sigma$ exclusion limits from mono- $h$, mono- $Z, t \bar{t}$ resonance (for scalar and pseudoscalar mediator) and Higgs to invisible searches (see text for details) in the $M_{a / S_{2}}-\tan \beta$ plane for the $2 \mathrm{HDM}+\mathrm{PS}$ (left) and $2 \mathrm{HDM}+\mathrm{S}$ (right). In both plots we use the parameters as given in eq. (4.4) and $M_{H / S_{1}}=M_{H^{ \pm}}=M_{A}=500 \mathrm{GeV}$.

\subsection{Combined constraints}

Finally, we summarize our results by comparing all derived limits for the two models in the $M_{a / S_{2}}-M_{A}$-plane and the $M_{a / S_{2}}-\tan \beta$-plane in figure 13 and figure 14, respectively. In addition to the $t \bar{t}$, mono- $Z$ and mono- $h$ constraints discussed above, we also include results from Higgs to invisible searches. The latest combined search by ATLAS [82] gives

$$
\mathrm{BR}(h \rightarrow \mathrm{inv})<0.26\left(0.17_{-0.05}^{+0.07}\right)
$$

for the observed (expected) upper limit at $95 \%$ confidence level. As $a$ and $S_{2}$ dominantly decay to DM, the 3-body final state $a / S_{2} \chi \chi$ is also invisible. Using the decay widths from section 2.4 we find a lower limit for $M_{a / S_{2}}$ of about $100 \mathrm{GeV}$ for $m_{\chi}=10 \mathrm{GeV}$ with a residual dependence on the mass of the heavy Higgs $M_{H / S_{1}}=M_{A}=M_{H^{ \pm}}$. This is also the reason why we start the parameter scans at $100 \mathrm{GeV}$ in terms of the light new Higgs mass $M_{a / S_{2}}$. 
The differences between the pseudoscalar and scalar model in the single searches have been discussed in the corresponding last sections, so here we focus on how the different constraints compare to each other within one model. Starting with the $2 \mathrm{HDM}+\mathrm{PS}$ model in the $M_{a}-M_{A}$-plane with $\tan \beta=1$, cf. left panel of figure 13 , we can see that the dominant limit comes from mono- $Z$ searches, excluding light Higgs masses up to $M_{a} \sim 320 \mathrm{GeV}$ and heavy Higgs masses between 200 and $1000 \mathrm{GeV}$, while for large masses of the heavy Higgses and small masses of the light Higgs the mono- $h$ limit leads to slightly stronger bounds. Light masses below $\sim 100 \mathrm{GeV}$ for the new pseudoscalar state are excluded by Higgs to invisible searches, while $t \bar{t}$ resonance searches can exclude small parts of the parameter space nearly independent of the light new pseudoscalar mass around $M_{H}=M_{H^{ \pm}}=M_{A} \sim 400$ and $500 \mathrm{GeV}$.

The same holds true for the $2 \mathrm{HDM}+\mathrm{S}$ model, cf. right panel of figure 13 , especially for the mono- $Z$ and Higgs to invisible searches. In contrast to that, the mono- $h$ limit for the scalar model is significantly weaker compared to the mono- $Z$ limits and thus never the strongest limit, as explained in section 4.4. Furthermore, the $t \bar{t}$ limits exclude a larger band of heavy Higgs masses from 400 to $500 \mathrm{GeV}$, however this is just a reflection of slightly stronger limits compared to the pseudoscalar case with the limits changing from just below $2 \sigma$ to just above $2 \sigma$.

For the $M_{a / S_{2}}-\tan \beta$-plane and at heavy Higgs masses of $500 \mathrm{GeV}$, cf. figure 14, we have again similar limits for both models, except for the mono- $h$ limit being weaker in the scalar model. As in the $M_{a}-M_{A}$-plane, the dominant limit is given by mono- $Z$ searches, specifically for values of $\tan \beta>1$. They begin to weaken for larger values of $\tan \beta$ of around three. While these limits, for $0.3 \lesssim \tan \beta \lesssim 3$ apply to 2 HDMs of all Yukawa sector types, cf. table 1 , for $\tan \beta \gtrsim 3$ the $b \bar{b}$ production mode starts to become relevant for type II and Y, leading to stronger limits for such Yukawa sectors, while for type I and $\mathrm{X} b \bar{b}$ production never becomes relevant, and limits will continue to get weaker for larger $\tan \beta$ values. The $t \bar{t}$ resonance searches provide a lower limit on $\tan \beta$ of around 1 , being slightly stronger in the $2 \mathrm{HDM}+\mathrm{S}$ and nearly independent of the light mediator mass, therefore being the strongest limit for $M_{a / S_{2}}>270 \mathrm{GeV}$.

Finally, another interesting aspect which is accessible via the comparison plots is the question of how to distinguish the two models. Here, the weaker mono- $h$ limits for the $2 \mathrm{HDM}+\mathrm{S}$ model can come in handy. This discrepancy in terms of sensitivity to the mono$h$ channel could be exploited to distinguish between the $2 \mathrm{HDM}+\mathrm{PS}$ and $2 \mathrm{HDM}+\mathrm{S}$, since the ratio of the signal strength in mono- $Z$ and mono- $h$ is characteristic for the model. So if signals are detected in both channels, their signal strength ratio could be used to discriminate between the models.

\section{Conclusions}

In this paper, we have investigated the collider phenomenology of the $2 \mathrm{HDM}+\mathrm{S}$ and $2 \mathrm{HDM}+\mathrm{PS}$, in particular focusing on the $t \bar{t}$, mono- $Z$ and mono- $h$ signatures.

The $2 \mathrm{HDM}+\mathrm{S}$ and $2 \mathrm{HDM}+\mathrm{PS}$ are the new standard paradigm used by the CMS and ATLAS collaborations to interpret experimental results in the context of DM searches and Simplified Models. These models feature an extended scalar sector, with a second Higgs 
doublet, and an additional singlet and differ from the previous generation of Simplified Models and other DM models by tending to generate a rich collider phenomenology. In particular, it is possible to have resonant production of the heavy Higgs bosons that can decay to a SM boson and the additional singlet, generating resonantly-enhanced mono- $Z$ and mono- $h$ signatures.

In our analysis, we made use of a few assumptions that have been established by the experimental collaborations [8] to reduce the number of dimensions of the parameter space, and simplify the comparison between the two models and the different experimental signatures. These assumptions are essentially the mass degeneracy of the heavy scalars, motivated by the bounds on the EW precision observables, together with the Higgs alignment limit, motivated by Higgs measurements. The alignment limit simplifies the Higgs sector by turning one of the doublets into the SM Higgs doublet, so that most constraints from Higgs physics are avoided. Moreover, while we have described the possible Yukawa sectors of the $2 \mathrm{HDM}$, our results are universal due to the range of parameters we considered.

We have reviewed all the principal constraints of the two models. Some of them, like Direct and Indirect Detection, are mostly complementary to collider searches, because they tend to require different mass spectra. Some other, like flavour and EW precision observables, perturbativity and unitarity constraints, instead are very relevant also for our signatures, and are used as a guidance to select the appropriate ranges for the various parameters.

Using the data provided by different LHC analyses, we derived limits for the $2 \mathrm{HDM}+\mathrm{S}$ and $2 \mathrm{HDM}+\mathrm{PS}$ for the $t \bar{t}$, mono- $Z$ and mono- $h$ signatures and discussed how they compare to each other and between the two models. We found that the mono- $Z$ limits are in general the most constraining ones, while the $t \bar{t}$ limits are nearly independent on the mass of the additional singlet and especially relevant for constraining $\tan \beta$. A lower bound of $\sim 100 \mathrm{GeV}$ for the additional singlet mass is given by Higgs to invisible searches, giving us a natural starting point for this parameter in our scans.

We found that in principle the two models could be discriminated at a collider by detecting both mono- $Z$ and mono- $h$ signatures, from the ratio of their strengths. Also, the absence or appearance of mono-jet signals would give further insights into the nature of the dark sector. However, depending on the DM mass, other probes, mostly astrophysical, would be powerful tools to help discriminating between the two models. A Direct Detection signal for DM is several orders of magnitude stronger for the $2 \mathrm{HDM}+\mathrm{S}$, while Indirect Detection signals would be several orders of magnitude stronger for the $2 \mathrm{HDM}+\mathrm{PS}$. So detecting one of these two astrophysical signatures would also give a clear indication towards the nature of the mediators to the dark sector and discriminate between $2 \mathrm{HDM}+\mathrm{S}$ and $2 \mathrm{HDM}+\mathrm{PS}$. In this case, collider studies could help to further investigate the inner workings of the dark sector.

\section{Acknowledgments}

VTT acknowledges support by the IMPRS-PTFS. The authors would like to thank Christopher Anelli, Dominick Cichon and Stefan Vogl for valuable discussions. Furthermore, we are grateful to Olivier Mattelaer of the MadGraph team for correspondence on the software. 


\section{A Formulae for the decay widths}

In this appendix we give analytic expressions for the dominant branching ratios of the spin-0 states in the $2 \mathrm{HDM}+\mathrm{S} / \mathrm{PS}$ as partially shown in section 2.4 .

We focus on the mass hierarchy $M_{A}, M_{H / S_{1}}, M_{H^{ \pm}}>M_{a / S_{2}}, M_{h}$ and $\tan \beta=\mathcal{O}(1)$. For the value of $\epsilon_{f}$, denoting the ratio between the Yukawa coupling of the fermion $f$ in the different types of $2 \mathrm{HDMs}$ and the SM value $y_{f}=\sqrt{2} m_{f} / v$, as given in table 1 . In the following we use the abbreviation $\tau_{i, j}=4 M_{i}^{2} / M_{j}^{2}$.

\section{A.1 Scalar model}

Higgs boson $\boldsymbol{h}$. As mentioned above, in the decoupling limit the couplings of $h$ with the SM states substantially coincide with the ones for the SM Higgs boson. However its total width can deviate, with respect to the SM prediction, because of the eventual presence of additional decay channels. The most relevant, if kinematically allowed, is the one into a pair of $S_{2}$ states. As the Higgs width is small, also three-body decays to $S_{2} \chi \bar{\chi}$ can be relevant and the additional widths are given by

$$
\begin{aligned}
\Gamma\left(h \rightarrow S_{2} S_{2}\right) & =\frac{1}{32 \pi} g_{h S_{2} S_{2}}^{2} M_{h} \sqrt{1-\tau_{S_{2}, h}}, \\
\Gamma\left(h \rightarrow S_{2} \chi \bar{\chi}\right) & =\frac{y_{\chi}^{2}}{32 \pi^{3}} g_{h S_{2} S_{2}}^{2} M_{h} g\left(\tau_{S_{2}, h}\right) \cos ^{2} \theta\left(1-\tau_{\chi, S_{2}}\right)^{3 / 2}, \\
\Gamma\left(h \rightarrow S_{2} f \bar{f}\right) & =\frac{N_{c}^{f} \epsilon_{f}^{2} y_{f}^{2}}{16 \pi^{3}} g_{h S_{2} S_{2}}^{2} M_{h} g\left(\tau_{S_{2}, h}\right) \sin ^{2} \theta\left(1-\tau_{f, S_{2}}\right)^{3 / 2},
\end{aligned}
$$

with [72]

$$
\begin{aligned}
g(\tau) & =\frac{\tau-4}{8}\left[4-\ln \left(\frac{\tau}{4}\right)\right]-\frac{5 \tau-4}{4 \sqrt{\tau-1}}\left[\arctan \left(\frac{\tau-2}{2 \sqrt{\tau-1}}\right)-\arctan \left(\frac{1}{\sqrt{\tau-1}}\right)\right], \\
g_{h S_{2} S_{2}} & =\frac{1}{M_{h} v}\left(M_{h}^{2}-2\left(M_{S_{1}}^{2}-M_{S_{2}}^{2}\right) \cos ^{2} \theta\right) \sin ^{2} \theta .
\end{aligned}
$$

Light scalar $\boldsymbol{S}_{\mathbf{2}}$. The light scalar $S_{2}$ mostly decays into $g g, f \bar{f}$ and $\chi \bar{\chi}$ (direct couplings with gauge boson are forbidden in the alignment limit), depending on the mass. We quote below the corresponding decay widths and the loop-induced one into gluons which is useful for the interpretation of the collider studies:

$$
\begin{aligned}
\Gamma\left(S_{2} \rightarrow g g\right) & =\frac{\alpha_{s}^{2}}{16 \pi^{3}} M_{S_{2}} \sin ^{2} \theta \sum_{q} \epsilon_{q}^{2} y_{q}^{2} F_{S}\left(\tau_{q, S_{2}}\right), \\
\Gamma\left(S_{2} \rightarrow f \bar{f}\right) & =\frac{N_{c}^{f} \epsilon_{f}^{2} y_{v}^{2}}{16 \pi} M_{S_{2}} \sin ^{2} \theta\left(1-\tau_{f, S_{2}}\right)^{3 / 2}, \\
\Gamma\left(S_{2} \rightarrow \chi \bar{\chi}\right) & =\frac{y_{\chi}^{2}}{8 \pi} M_{S_{2}} \cos ^{2} \theta\left(1-\tau_{\chi, S_{2}}\right)^{3 / 2},
\end{aligned}
$$

with

$$
F_{S}(x)=x\left|1+(1-x) \arctan ^{2} \frac{1}{\sqrt{x-1}}\right|^{2}
$$


Heavy scalar $\boldsymbol{S}_{\mathbf{1}}$. Besides an additional $\cos ^{2} \theta$ due to the mixing with the additional singlet the couplings of the heavy scalar to SM fields remain similar to the ones known from 2 HDMs. Additional decay channels are $\chi \bar{\chi}$, suppressed by $\sin ^{2} \theta, S_{2} S_{2}$, which is very small for our parameter choice and $h S_{2}$ which is important for the mono- $h$ bounds. The analytic expressions are given by

$$
\begin{aligned}
\Gamma\left(S_{1} \rightarrow g g\right) & =\frac{\alpha_{s}^{2}}{16 \pi^{3}} M_{S_{1}} \cos ^{2} \theta \sum_{q} \epsilon_{q}^{2} y_{q}^{2} F_{S}\left(\tau_{q, S_{1}}\right), \\
\Gamma\left(S_{1} \rightarrow f \bar{f}\right) & =\frac{N_{c}^{f} \epsilon_{f}^{2} y_{f}^{2}}{16 \pi} M_{S_{1}} \cos ^{2} \theta\left(1-\tau_{f, S_{1}}\right)^{3 / 2}, \\
\Gamma\left(S_{1} \rightarrow \chi \bar{\chi}\right) & =\frac{y_{\chi}^{2}}{8 \pi} M_{S_{1}} \sin ^{2} \theta\left(1-\tau_{\chi, S_{1}}\right)^{3 / 2}, \\
\Gamma\left(S_{1} \rightarrow S_{2} S_{2}\right) & =\frac{1}{32 \pi} g_{S_{1} S_{2} S_{2}}^{2} M_{S_{1}} \sqrt{1-\tau_{S_{2}, S_{1}}}, \\
\Gamma\left(S_{1} \rightarrow S_{2} h\right) & =\frac{1}{16 \pi} \frac{\lambda^{1 / 2}\left(M_{S_{1}}, M_{h}, M_{S_{2}}\right)}{M_{S_{1}}} g_{S_{1} h S_{2}}^{2},
\end{aligned}
$$

with

$$
\begin{aligned}
g_{S_{1} S_{2} S_{2}} & =\frac{1}{M_{S_{1}} v_{S}}\left(M_{S_{1}}^{2}+2 M_{S_{2}}^{2}-\frac{2-3 \sin ^{2} \theta}{\cos ^{2} \theta} \hat{\lambda}_{H H S} v_{S}^{2}\right) \sin \theta \cos \theta \\
g_{S_{1} h S_{2}} & =\frac{1}{M_{S_{1}} v}\left(M_{h}^{2}+\left(M_{S_{1}}^{2}-M_{S_{2}}^{2}\right) \cos 2 \theta\right) \sin \theta \cos \theta
\end{aligned}
$$

Furthermore, we have introduced

$$
\lambda\left(m_{1}, m_{2}, m_{3}\right)=\left(m_{1}^{2}-m_{2}^{2}-m_{3}^{2}\right)^{2}-4 m_{2}^{2} m_{3}^{2} .
$$

Pseudoscalar $\boldsymbol{A}$. Besides the partial widths known from 2HDMs the heavy pseudoscalar has an additional decay channel to $S_{2} Z$, which are given by

$$
\begin{aligned}
\Gamma(A \rightarrow g g) & =\frac{\alpha_{s}^{2}}{16 \pi^{3}} M_{A} \sum_{q} \epsilon_{q}^{2} y_{q}^{2} F_{P}\left(\tau_{q, A}\right), \\
\Gamma(A \rightarrow f \bar{f}) & =\frac{N_{c}^{f} \epsilon_{f}^{2} y_{f}^{2}}{16 \pi} M_{A}\left(1-\tau_{f, A}\right)^{1 / 2}, \\
\Gamma\left(A \rightarrow S_{2} Z\right) & =\frac{1}{16 \pi} \frac{\lambda^{3 / 2}\left(M_{A}, M_{S_{2}}, M_{Z}\right)}{M_{A}^{3} v^{2}},
\end{aligned}
$$

with

$$
F_{P}(x)=x\left|\arctan ^{2} \frac{1}{\sqrt{x-1}}\right|^{2}
$$

Charged scalar $\boldsymbol{H}^{ \pm}$. For completeness also the partial widths of $H^{ \pm}$to quarks and the new spin- 0 state plus a $W^{ \pm}$, since the $H^{+} h W^{+}$vertex vanishes in the alignment limit, are 
given by

$$
\begin{aligned}
\Gamma\left(H^{+} \rightarrow t \bar{b}\right) & =\frac{N_{c}^{t}\left|V_{t b}\right|^{2} \epsilon_{t}^{2} y_{t}^{2}}{16 \pi} M_{H^{ \pm}}\left(1-\tau_{t, H^{ \pm}} / 4\right)^{2}, \\
\Gamma\left(H^{ \pm} \rightarrow S_{1} W^{ \pm}\right) & =\frac{1}{16 \pi} \frac{\lambda^{3 / 2}\left(M_{H^{ \pm}}, M_{S_{1}}, M_{W}\right)}{M_{H^{ \pm}}^{3} v^{2}} \cos ^{2} \theta, \\
\Gamma\left(H^{ \pm} \rightarrow A W^{ \pm}\right) & =\frac{1}{16 \pi} \frac{\lambda^{3 / 2}\left(M_{H^{ \pm}}, M_{A}, M_{W}\right)}{M_{H^{ \pm}}^{3} v^{2}}, \\
\Gamma\left(H^{ \pm} \rightarrow S_{2} W^{ \pm}\right) & =\frac{1}{16 \pi} \frac{\lambda^{3 / 2}\left(M_{H^{ \pm}}, M_{S_{2}}, M_{W}\right)}{M_{H^{ \pm}}^{3} v^{2}} \sin ^{2} \theta,
\end{aligned}
$$

where in the case of $H^{+} \rightarrow t \bar{b}$ we have neglected terms of $\mathcal{O}\left(m_{b}^{2} / M_{H^{ \pm}}^{2}\right)$.

\section{A.2 Pseudoscalar model}

The reults in this section are taken from [3] and transferred to our notation. The features are very similar to the ones in the previous section.

Higgs boson $\boldsymbol{h}$. Similarly to the $2 \mathrm{HDM}+\mathrm{S}$ the couplings of $h$ to $\bar{f} f$ and gauge boson pairs are kept to their SM values by the alignment limit. Its total width might be nevertheless enlarged by additional two and/or three body decay channels being [72]:

$$
\begin{aligned}
\Gamma(h \rightarrow a a) & =\frac{1}{32 \pi} g_{\text {haa }}^{2} M_{h}\left(1-\tau_{a, h}\right)^{1 / 2}, \\
\Gamma(h \rightarrow a \chi \bar{\chi}) & =\frac{y_{\chi}^{2}}{32 \pi^{3}} g_{h a a}^{2} M_{h} g\left(\tau_{a, h}\right) \cos ^{2} \theta\left(1-\tau_{\chi, a}\right)^{1 / 2}, \\
\Gamma(h \rightarrow a f \bar{f}) & =\frac{N_{c}^{f} \epsilon_{f}^{2} y_{f}^{2}}{16 \pi^{3}} g_{h a a}^{2} M_{h} g\left(\tau_{a, h}\right) \sin ^{2} \theta\left(1-\tau_{f, a}\right)^{1 / 2},
\end{aligned}
$$

with $g(\tau)$ given in eq. (A.4) and

$$
\begin{aligned}
g_{h a a}=\frac{1}{M_{h} v}[ & \left(M_{h}^{2}-2 M_{H}^{2}+4 M_{H^{ \pm}}^{2}-2 M_{a}^{2}-2 \lambda_{3} v^{2}\right) \sin ^{2} \theta \\
& \left.-\left(\lambda_{11 P} \cos ^{2} \beta+\lambda_{22 P} \sin ^{2} \beta\right) v^{2} \cos ^{2} \theta\right] .
\end{aligned}
$$

Light pseudoscalar $a$. The partial widths to $g g$ and $f \bar{f}$ and $\chi \bar{\chi}$ are given by

$$
\begin{aligned}
& \Gamma(a \rightarrow g g)=\frac{\alpha_{s}^{2}}{16 \pi^{3}} M_{a} \sin ^{2} \theta \sum_{q} \epsilon_{q}^{2} y_{q}^{2} F_{P}\left(\tau_{q, A}\right), \\
& \Gamma(a \rightarrow f \bar{f})=\frac{N_{c}^{f} \epsilon_{f}^{2} y_{f}^{2}}{16 \pi} M_{a} \sin ^{2} \theta\left(1-\tau_{f, a}\right)^{1 / 2}, \\
& \Gamma(a \rightarrow \chi \bar{\chi})=\frac{y_{\chi}^{2}}{8 \pi} M_{a} \cos ^{2} \theta\left(1-\tau_{\chi, a}\right)^{1 / 2},
\end{aligned}
$$

where $F_{P}$ is given in eq. (A.21). 
Heavy pseudoscalar $A$. The partial widths to $g g$ and $f \bar{f}, \chi \bar{\chi}$ and $a h$ are given by

$$
\begin{aligned}
\Gamma(A \rightarrow g g) & =\frac{\alpha_{s}^{2}}{16 \pi^{3}} M_{A} \cos ^{2} \theta \sum_{q} \epsilon_{q}^{2} y_{q}^{2} F_{P}\left(\tau_{q, A}\right), \\
\Gamma(A \rightarrow f \bar{f}) & =\frac{N_{c}^{f} \epsilon_{f}^{2} y_{f}^{2}}{16 \pi} M_{A} \cos ^{2} \theta\left(1-\tau_{f, A}\right)^{1 / 2}, \\
\Gamma(A \rightarrow \chi \bar{\chi}) & =\frac{y_{\chi}^{2}}{8 \pi} M_{A} \sin ^{2} \theta\left(1-\tau_{\chi, A}\right)^{1 / 2}, \\
\Gamma(A \rightarrow a h) & =\frac{1}{16 \pi} \frac{\lambda^{1 / 2}\left(M_{A}, M_{a}, M_{h}\right)}{M_{A}} g_{\text {Aah }}^{2},
\end{aligned}
$$

with

$$
\begin{aligned}
g_{A a h}=\frac{1}{M_{A} v} & {\left[M_{h}^{2}-2 M_{H}^{2}-M_{A}^{2}+4 M_{H^{ \pm}}^{2}-M_{a}^{2}\right.} \\
& \left.+\left(\lambda_{11 P} \cos ^{2} \beta+\lambda_{22 P} \sin ^{2} \beta-2 \lambda_{3}\right) v^{2}\right] \sin \theta \cos \theta .
\end{aligned}
$$

Heavy scalar $H$. The partial widths to $g g$ and $f \bar{f}, a a$ and $a Z$ are given by

$$
\begin{aligned}
\Gamma(H \rightarrow g g) & =\frac{\alpha_{s}^{2}}{16 \pi^{3}} M_{H} \sum_{q} \epsilon_{q}^{2} y_{q}^{2} F_{S}\left(\tau_{q, H}\right), \\
\Gamma(H \rightarrow f \bar{f}) & =\frac{N_{c}^{f} \epsilon_{f}^{2} y_{f}^{2}}{16 \pi} M_{H}\left(1-\tau_{f, H}\right)^{3 / 2}, \\
\Gamma(H \rightarrow a a) & =\frac{1}{32 \pi} g_{H a a}^{2} M_{H}\left(1-\tau_{a, H}\right)^{1 / 2}, \\
\Gamma(H \rightarrow a Z) & =\frac{1}{16 \pi} \frac{\lambda^{3 / 2}\left(M_{H}, M_{a}, M_{Z}\right)}{M_{H}^{3} v^{2}} \sin ^{2} \theta,
\end{aligned}
$$

with

$$
\begin{aligned}
g_{H a a}=\frac{1}{M_{H} v} & {\left[\cot (2 \beta)\left(2 M_{h}^{2}-4 M_{H}^{2}+4 M_{H^{ \pm}}^{2}-2 \lambda_{3} v^{2}\right) \sin ^{2} \theta\right.} \\
& \left.+\sin (2 \beta) \cos ^{2} \theta v^{2}\left(\lambda_{11 P}-\lambda_{22 P}\right) / 2\right]
\end{aligned}
$$

denoting the Haa coupling and $\lambda$ is given in eq. (A.17).

Charged scalar $H^{ \pm}$. Since in the alignment limit the $H^{+} h W^{+}$vertex vanishes, the partial decay widths of the charged scalar $H^{ \pm}$relevant for small $\tan \beta$ are given by

$$
\begin{aligned}
\Gamma\left(H^{+} \rightarrow t \bar{b}\right) & =\frac{N_{c}^{t}\left|V_{t b}\right|^{2} \epsilon_{t}^{2} y_{t}^{2}}{16 \pi} M_{H^{ \pm}}\left(1-\tau_{t, H^{ \pm}} / 4\right)^{2}, \\
\Gamma\left(H^{ \pm} \rightarrow H W^{ \pm}\right) & =\frac{1}{16 \pi} \frac{\lambda^{3 / 2}\left(M_{H^{ \pm}}, M_{H}, M_{W}\right)}{M_{H^{ \pm}}^{3} v^{2}}, \\
\Gamma\left(H^{ \pm} \rightarrow A W^{ \pm}\right) & =\frac{1}{16 \pi} \frac{\lambda^{3 / 2}\left(M_{H^{ \pm}}, M_{A}, M_{W}\right)}{M_{H^{ \pm}}^{3} v^{2}} \cos ^{2} \theta, \\
\Gamma\left(H^{ \pm} \rightarrow a W^{ \pm}\right) & =\frac{1}{16 \pi} \frac{\lambda^{3 / 2}\left(M_{H^{ \pm}}, M_{a}, M_{W}\right)}{M_{H^{ \pm}}^{3} v^{2}} \sin ^{2} \theta,
\end{aligned}
$$

where in the case of $H^{+} \rightarrow t \bar{b}$ we have neglected terms of $\mathcal{O}\left(m_{b}^{2} / M_{H^{ \pm}}^{2}\right)$ again. 
Open Access. This article is distributed under the terms of the Creative Commons Attribution License (CC-BY 4.0), which permits any use, distribution and reproduction in any medium, provided the original author(s) and source are credited.

\section{References}

[1] J. Abdallah et al., Simplified Models for Dark Matter and Missing Energy Searches at the $L H C$, arXiv:1409.2893 [INSPIRE].

[2] G. Arcadi et al., The waning of the WIMP? A review of models, searches and constraints, Eur. Phys. J. C 78 (2018) 203 [arXiv:1703.07364] [InSPIRE].

[3] M. Bauer, U. Haisch and F. Kahlhoefer, Simplified dark matter models with two Higgs doublets: I. Pseudoscalar mediators, JHEP 05 (2017) 138 [arXiv:1701.07427] [INSPIRE].

[4] ATLAS collaboration, Search for dark matter and other new phenomena in events with an energetic jet and large missing transverse momentum using the ATLAS detector, JHEP 01 (2018) 126 [arXiv: 1711.03301] [INSPIRE].

[5] CMS collaboration, Search for new physics in final states with an energetic jet or a hadronically decaying $W$ or $Z$ boson and transverse momentum imbalance at $\sqrt{s}=13 \mathrm{TeV}$, Phys. Rev. D 97 (2018) 092005 [arXiv:1712.02345] [INSPIRE].

[6] J.M. No, Looking through the pseudoscalar portal into dark matter: Novel mono-Higgs and mono-Z signatures at the LHC, Phys. Rev. D 93 (2016) 031701 [arXiv:1509.01110] [INSPIRE].

[7] N.F. Bell, G. Busoni and I.W. Sanderson, Two Higgs Doublet Dark Matter Portal, JCAP 01 (2018) 015 [arXiv: 1710.10764] [INSPIRE].

[8] LHC Dark Matter Working Group collaboration, LHC Dark Matter Working Group: Next-generation spin-0 dark matter models, Phys. Dark Univ. 27 (2020) 100351 [arXiv: 1810.09420] [INSPIRE].

[9] S. von Buddenbrock et al., Phenomenological signatures of additional scalar bosons at the LHC, Eur. Phys. J. C 76 (2016) 580 [arXiv:1606.01674] [INSPIRE].

[10] S. von Buddenbrock et al., Constraints on a 2HDM with a singlet scalar and implications in the search for heavy bosons at the LHC, J. Phys. G 46 (2019) 115001 [arXiv:1809.06344] [INSPIRE].

[11] S. Baum, K. Freese, N.R. Shah and B. Shakya, NMSSM Higgs boson search strategies at the LHC and the mono-Higgs signature in particular, Phys. Rev. D 95 (2017) 115036 [arXiv: 1703.07800] [INSPIRE].

[12] S. Baum and N.R. Shah, Two Higgs Doublets and a Complex Singlet: Disentangling the Decay Topologies and Associated Phenomenology, arXiv:1808.02667 [InSPIRE].

[13] S. Baum and N.R. Shah, Benchmark Suggestions for Resonant Double Higgs Production at the LHC for Extended Higgs Sectors, arXiv:1904.10810 [INSPIRE].

[14] F. Kahlhoefer, K. Schmidt-Hoberg, T. Schwetz and S. Vogl, Implications of unitarity and gauge invariance for simplified dark matter models, JHEP 02 (2016) 016 [arXiv: 1510.02110] [INSPIRE].

[15] N.F. Bell, Y. Cai and R.K. Leane, Impact of mass generation for spin-1 mediator simplified models, JCAP 01 (2017) 039 [arXiv:1610.03063] [INSPIRE]. 
[16] N.F. Bell, G. Busoni and I.W. Sanderson, Self-consistent Dark Matter Simplified Models with an s-channel scalar mediator, JCAP 03 (2017) 015 [arXiv: 1612.03475] [INSPIRE].

[17] C. Englert, M. McCullough and M. Spannowsky, S-Channel Dark Matter Simplified Models and Unitarity, Phys. Dark Univ. 14 (2016) 48 [arXiv:1604.07975] [INSPIRE].

[18] D. Goncalves, P.A.N. Machado and J.M. No, Simplified Models for Dark Matter Face their Consistent Completions, Phys. Rev. D 95 (2017) 055027 [arXiv:1611.04593] [INSPIRE].

[19] S. Ipek, D. McKeen and A.E. Nelson, A Renormalizable Model for the Galactic Center Gamma Ray Excess from Dark Matter Annihilation, Phys. Rev. D 90 (2014) 055021 [arXiv:1404.3716] [INSPIRE].

[20] N.F. Bell, G. Busoni and I.W. Sanderson, Loop Effects in Direct Detection, JCAP 08 (2018) 017 [Erratum ibid. 01 (2019) E01] [arXiv: 1803.01574] [INSPIRE].

[21] G. Arcadi, M. Lindner, F.S. Queiroz, W. Rodejohann and S. Vogl, Pseudoscalar Mediators: A WIMP model at the Neutrino Floor, JCAP 03 (2018) 042 [arXiv: 1711. 02110] [INSPIRE].

[22] G. Arcadi, 2HDM portal for Singlet-Doublet Dark Matter, Eur. Phys. J. C 78 (2018) 864 [arXiv: 1804.04930] [INSPIRE].

[23] S. Davidson and H.E. Haber, Basis-independent methods for the two-Higgs-doublet model, Phys. Rev. D 72 (2005) 035004 [Erratum ibid. D 72 (2005) 099902] [hep-ph/0504050] [INSPIRE].

[24] A. Trautner, Systematic construction of basis invariants in the 2HDM, JHEP 05 (2019) 208 [arXiv: 1812.02614] [INSPIRE].

[25] I.P. Ivanov, C.C. Nishi and A. Trautner, Beyond basis invariants, Eur. Phys. J. C 79 (2019) 315 [arXiv: 1901.11472] [INSPIRE].

[26] P.S. Bhupal Dev and A. Pilaftsis, Maximally Symmetric Two Higgs Doublet Model with Natural Standard Model Alignment, JHEP 12 (2014) 024 [Erratum ibid. 11 (2015) 147] [arXiv: 1408.3405] [INSPIRE].

[27] A. Pich and P. Tuzon, Yukawa Alignment in the Two-Higgs-Doublet Model, Phys. Rev. D 80 (2009) 091702 [arXiv:0908.1554] [INSPIRE].

[28] P. Tuzon and A. Pich, The Aligned two-Higgs Doublet model, Acta Phys. Polon. Supp. 3 (2010) 215 [arXiv: 1001.0293] [INSPIRE].

[29] A. Pich, Flavour constraints on multi-Higgs-doublet models: Yukawa alignment, Nucl. Phys. Proc. Suppl. 209 (2010) 182 [arXiv:1010.5217] [INSPIRE].

[30] A. Peñuelas and A. Pich, Flavour alignment in multi-Higgs-doublet models, JHEP 12 (2017) 084 [arXiv: 1710.02040 ] [INSPIRE].

[31] S. Gori, H.E. Haber and E. Santos, High scale flavor alignment in two-Higgs doublet models and its phenomenology, JHEP 06 (2017) 110 [arXiv:1703.05873] [INSPIRE].

[32] W. Rodejohann and U. Saldaña-Salazar, Multi-Higgs-Doublet Models and Singular Alignment, JHEP 07 (2019) 036 [arXiv: 1903. 00983] [INSPIRE].

[33] S. Centelles Chuliá, W. Rodejohann and U.J. Saldaña-Salazar, Two-Higgs-doublet models with a flavored $\mathbb{Z}_{2}$ symmetry, Phys. Rev. D 101 (2020) 035013 [arXiv:1911.06824] [INSPIRE].

[34] M. Cirelli, E. Del Nobile and P. Panci, Tools for model-independent bounds in direct dark matter searches, JCAP 10 (2013) 019 [arXiv:1307.5955] [INSPIRE]. 
[35] M.J. Dolan, F. Kahlhoefer, C. McCabe and K. Schmidt-Hoberg, A taste of dark matter: Flavour constraints on pseudoscalar mediators, JHEP 03 (2015) 171 [Erratum ibid. 07 (2015) 103] [arXiv: 1412.5174] [INSPIRE].

[36] C. Arina, E. Del Nobile and P. Panci, Dark Matter with Pseudoscalar-Mediated Interactions Explains the DAMA Signal and the Galactic Center Excess, Phys. Rev. Lett. 114 (2015) 011301 [arXiv: 1406 .5542] [INSPIRE].

[37] T. Abe, M. Fujiwara and J. Hisano, Loop corrections to dark matter direct detection in a pseudoscalar mediator dark matter model, JHEP 02 (2019) 028 [arXiv:1810.01039] [INSPIRE].

[38] F. Ertas and F. Kahlhoefer, Loop-induced direct detection signatures from CP-violating scalar mediators, JHEP 06 (2019) 052 [arXiv:1902.11070] [INSPIRE].

[39] T. Abe, M. Fujiwara, J. Hisano and Y. Shoji, Maximum value of the spin-independent cross section in the 2HDM+a, JHEP 01 (2020) 114 [arXiv: 1910.09771] [INSPIRE].

[40] M. Freytsis and Z. Ligeti, On dark matter models with uniquely spin-dependent detection possibilities, Phys. Rev. D 83 (2011) 115009 [arXiv:1012.5317] [INSPIRE].

[41] G. Arcadi, A. Djouadi and M. Raidal, Dark Matter through the Higgs portal, Phys. Rept. 842 (2020) 1 [arXiv: 1903.03616] [INSPIRE].

[42] Fermi-LAT and DES collaborations, Searching for Dark Matter Annihilation in Recently Discovered Milky Way Satellites with Fermi-LAT, Astrophys. J. 834 (2017) 110 [arXiv: 1611.03184] [INSPIRE].

[43] T. Enomoto and R. Watanabe, Flavor constraints on the Two Higgs Doublet Models of $Z_{2}$ symmetric and aligned types, JHEP 05 (2016) 002 [arXiv:1511.05066] [INSPIRE].

[44] H.E. Haber and A. Pomarol, Constraints from global symmetries on radiative corrections to the Higgs sector, Phys. Lett. B 302 (1993) 435 [hep-ph/9207267] [InSPIRE].

[45] A. Pomarol and R. Vega, Constraints on CP-violation in the Higgs sector from the rho parameter, Nucl. Phys. B 413 (1994) 3 [hep-ph/9305272] [INSPIRE].

[46] R. Barbieri, L.J. Hall and V.S. Rychkov, Improved naturalness with a heavy Higgs: An Alternative road to LHC physics, Phys. Rev. D 74 (2006) 015007 [hep-ph/0603188] [INSPIRE].

[47] J.M. Gerard and M. Herquet, A Twisted custodial symmetry in the two-Higgs-doublet model, Phys. Rev. Lett. 98 (2007) 251802 [hep-ph/0703051] [INSPIRE].

[48] B. Grzadkowski, M. Maniatis and J. Wudka, The bilinear formalism and the custodial symmetry in the two-Higgs-doublet model, JHEP 11 (2011) 030 [arXiv:1011.5228] [INSPIRE].

[49] H.E. Haber and D. O'Neil, Basis-independent methods for the two-Higgs-doublet model III: The CP-conserving limit, custodial symmetry and the oblique parameters $S, T, U$, Phys. Rev. D 83 (2011) 055017 [arXiv:1011.6188] [INSPIRE].

[50] S. Kanemura and K. Yagyu, Unitarity bound in the most general two Higgs doublet model, Phys. Lett. B 751 (2015) 289 [arXiv:1509.06060] [INSPIRE].

[51] K.G. Klimenko, On Necessary and Sufficient Conditions for Some Higgs Potentials to Be Bounded From Below, Theor. Math. Phys. 62 (1985) 58 [INSPIRE]. 
[52] ATLAS collaboration, Search for dark matter produced in association with bottom or top quarks in $\sqrt{s}=13 \mathrm{TeV}$ pp collisions with the ATLAS detector, Eur. Phys. J. C 78 (2018) 18 [arXiv: 1710.11412] [INSPIRE].

[53] CMS collaboration, Search for dark matter produced in association with a single top quark or a top quark pair in proton-proton collisions at $\sqrt{s}=13$ TeV, JHEP 03 (2019) 141 [arXiv: 1901.01553] [INSPIRE].

[54] ATLAS collaboration, Constraints on mediator-based dark matter and scalar dark energy models using $\sqrt{s}=13 \mathrm{TeV}$ pp collision data collected by the ATLAS detector, JHEP 05 (2019) 142 [arXiv: 1903.01400] [INSPIRE].

[55] E. Bernreuther, J. Horak, T. Plehn and A. Butter, Actual Physics behind Mono-X, SciPost Phys. 5 (2018) 034 [arXiv:1805.11637] [InSPIRE].

[56] D. Dercks, N. Desai, J.S. Kim, K. Rolbiecki, J. Tattersall and T. Weber, CheckMATE 2: From the model to the limit, Comput. Phys. Commun. 221 (2017) 383 [arXiv:1611.09856] [INSPIRE].

[57] F. D'Eramo, J. de Vries and P. Panci, A $750 \mathrm{GeV}$ Portal: LHC Phenomenology and Dark Matter Candidates, JHEP 05 (2016) 089 [arXiv:1601.01571] [INSPIRE].

[58] J.F. Gunion, H.E. Haber, G.L. Kane and S. Dawson, The Higgs Hunter's Guide, Front. Phys. 80 (2000) 1 [INSPIRE].

[59] J. Alwall et al., The automated computation of tree-level and next-to-leading order differential cross sections and their matching to parton shower simulations, JHEP 07 (2014) 079 [arXiv: 1405.0301] [INSPIRE].

[60] V. Hirschi and O. Mattelaer, Automated event generation for loop-induced processes, JHEP 10 (2015) 146 [arXiv: 1507.00020] [inSPIRE].

[61] G. Ossola, C.G. Papadopoulos and R. Pittau, CutTools: A Program implementing the OPP reduction method to compute one-loop amplitudes, JHEP 03 (2008) 042 [arXiv:0711.3596] [INSPIRE].

[62] T. Peraro, Ninja: Automated Integrand Reduction via Laurent Expansion for One-Loop Amplitudes, Comput. Phys. Commun. 185 (2014) 2771 [arXiv:1403.1229] [InSPIRE].

[63] A. Denner, S. Dittmaier and L. Hofer, Collier: a fortran-based Complex One-Loop LIbrary in Extended Regularizations, Comput. Phys. Commun. 212 (2017) 220 [arXiv:1604.06792] [INSPIRE].

[64] J. Butterworth et al., PDF 4 LHC recommendations for LHC Run II, J. Phys. G 43 (2016) 023001 [arXiv: 1510.03865 ] [INSPIRE].

[65] A. Buckley et al., LHAPDF6: parton density access in the LHC precision era, Eur. Phys. J. C 75 (2015) 132 [arXiv: 1412.7420] [INSPIRE].

[66] T. Sjöstrand et al., An Introduction to PYTHIA 8.2, Comput. Phys. Commun. 191 (2015) 159 [arXiv: 1410.3012] [INSPIRE].

[67] DELPHES 3 collaboration, DELPHES 3, A modular framework for fast simulation of a generic collider experiment, JHEP 02 (2014) 057 [arXiv:1307.6346] [INSPIRE].

[68] E. Conte and B. Fuks, Confronting new physics theories to LHC data with MADANALYSIS 5, Int. J. Mod. Phys. A 33 (2018) 1830027 [arXiv:1808.00480] [InSPIRE]. 
[69] E. Conte, B. Dumont, B. Fuks and C. Wymant, Designing and recasting LHC analyses with MadAnalysis 5, Eur. Phys. J. C 74 (2014) 3103 [arXiv:1405.3982] [InSPIRE].

[70] ATLAS collaboration, Search for Heavy Higgs Bosons A/H Decaying to a Top Quark Pair in pp Collisions at $\sqrt{s}=8 \mathrm{TeV}$ with the ATLAS Detector, Phys. Rev. Lett. 119 (2017) 191803 [arXiv: 1707.06025] [INSPIRE].

[71] CMS collaboration, Search for heavy Higgs bosons decaying to a top quark pair in proton-proton collisions at $\sqrt{s}=13 \mathrm{TeV}$, JHEP 04 (2020) 171 [arXiv:1908.01115] [INSPIRE].

[72] A. Djouadi, J. Kalinowski and P.M. Zerwas, Two and three-body decay modes of SUSY Higgs particles, Z. Phys. C 70 (1996) 435 [hep-ph/9511342] [INSPIRE].

[73] T. Hahn, S. Heinemeyer, F. Maltoni, G. Weiglein and S. Willenbrock, SM and MSSM Higgs boson production cross-sections at the Tevatron and the LHC, in TEV4LHC Workshop: 3rd Meeting, Geneva, Switzerland, 28-30 April 2005 (2006) [hep-ph/0607308] [INSPIRE].

[74] M. Bauer, M. Klassen and V. Tenorth, Universal properties of pseudoscalar mediators in dark matter extensions of 2HDMs, JHEP 07 (2018) 107 [arXiv: 1712.06597] [INSPIRE].

[75] ATLAS collaboration, Search for an invisibly decaying Higgs boson or dark matter candidates produced in association with a $Z$ boson in pp collisions at $\sqrt{s}=13 \mathrm{TeV}$ with the ATLAS detector, Phys. Lett. B 776 (2018) 318 [arXiv:1708.09624] [INSPIRE].

[76] CMS collaboration, Search for new physics in events with a leptonically decaying $Z$ boson and a large transverse momentum imbalance in proton-proton collisions at $\sqrt{s}=13 \mathrm{TeV}$, Eur. Phys. J. C 78 (2018) 291 [arXiv:1711.00431] [INSPIRE].

[77] G. Cowan, Discovery sensitivity for a counting experiment with background uncertainty, available at https://www.pp.rhul.ac.uk/ cowan/stat/medsig/medsigNote.pdf.

[78] CMS collaboration, Projection of the Mono-Z search for dark matter to the HL-LHC, CMS-PAS-FTR-18-007.

[79] ATLAS collaboration, Search for Dark Matter Produced in Association with a Higgs Boson Decaying to $b \bar{b}$ using $36 \mathrm{fb}^{-1}$ of pp collisions at $\sqrt{s}=13 \mathrm{TeV}$ with the ATLAS Detector, Phys. Rev. Lett. 119 (2017) 181804 [arXiv:1707.01302] [INSPIRE].

[80] CMS collaboration, Search for dark matter produced in association with a Higgs boson decaying to a pair of bottom quarks in proton-proton collisions at $\sqrt{s}=13 \mathrm{TeV}$, Eur. Phys. J. C 79 (2019) 280 [arXiv:1811.06562] [INSPIRE].

[81] CMS collaboration, Search for dark matter particles produced in association with a Higgs boson in proton-proton collisions at $\sqrt{\mathrm{s}}=13 \mathrm{TeV}$, JHEP 03 (2020) 025 [arXiv: 1908.01713] [INSPIRE].

[82] ATLAS collaboration, Combination of searches for invisible Higgs boson decays with the ATLAS experiment, Phys. Rev. Lett. 122 (2019) 231801 [arXiv:1904.05105] [INSPIRE]. 